\title{
GENERALIZED BERGMAN KERNELS ON SYMPLECTIC MANIFOLDS OF BOUNDED GEOMETRY
}

\author{
YURI A. KORDYUKOV, XIAONAN MA, AND GEORGE MARINESCU
}

\begin{abstract}
We study the asymptotic behavior of the generalized Bergman kernel of the renormalized Bochner-Laplacian on high tensor powers of a positive line bundle on a symplectic manifold of bounded geometry. First, we establish the off-diagonal exponential estimate for the generalized Bergman kernel. As an application, we obtain the relation between the generalized Bergman kernel on a Galois covering of a compact symplectic manifold and the generalized Bergman kernel on the base. Then we state the full off-diagonal asymptotic expansion of the generalized Bergman kernel, improving the remainder estimate known in the compact case to an exponential decay. Finally, we establish the theory of Berezin-Toeplitz quantization on symplectic orbifolds associated with the renormalized Bochner-Laplacian.
\end{abstract}

\section{CONTENTS}

1. Introduction

2. Preliminaries on differential operators and Sobolev spaces

2.1. Differential operators

2.2. Sobolev spaces

2.3. Sobolev embedding theorem

2.4. The renormalized Bochner-Laplacian

3. Proof of main results

3.1. Weight functions

3.2. Weighted estimates for the renormalized Bochner-Laplacian

3.3. Weighted estimates for the resolvent

3.4. Pointwise exponential estimates for the resolvents

3.5. Proof of Theorems 1.2 and 1.3

4. Full off-diagonal asymptotic expansion

5. Berezin-Toeplitz quantization on orbifolds

5.1. Basic definitions on orbifolds

5.2. Bergman kernel on symplectic orbifolds

5.3. Berezin-Toeplitz quantization on symplectic orbifolds

2000 Mathematics Subject Classification. Primary 58J37; Secondary 53D05.

Key words and phrases. Bergman kernel, Bochner-Laplacian, symplectic manifold, bounded geometry, Berezin-Toeplitz quantization, orbifold.

Y. K. supported by the Russian Science Foundation, project no. 17-11-01004.

X. M. partially supported by NNSFC 11528103, ANR-14-CE25-0012-01 and funded through the Institutional Strategy of the University of Cologne within the German Excellence Initiative.

G. M. partially supported by DFG funded project SFB TRR 191. 


\section{INTRODUCTION}

In this article, we consider a smooth symplectic manifold $(X, \omega)$ of dimension $2 n$. Let $\left(L, h^{L}\right)$ be a Hermitian line bundle on $X$ with a Hermitian connection $\nabla^{L}: \mathscr{C}^{\infty}(X, L) \rightarrow$ $\mathscr{C}^{\infty}\left(X, T^{*} X \otimes L\right)$. We assume that $L$ satisfies the prequantization condition:

$$
\frac{i}{2 \pi} R^{L}=\omega
$$

where $R^{L}=\left(\nabla^{L}\right)^{2}$ is the curvature of the connection $\nabla^{L}$. Let $\left(E, h^{E}\right)$ be a Hermitian vector bundle on $X$ with Hermitian connection $\nabla^{E}$ and its curvature $R^{E}$.

Let $g^{T X}$ be a Riemannian metric on $X$ and $\nabla^{T X}$ be the Levi-Civita connection of $\left(X, g^{T X}\right)$. Let $J_{0}: T X \rightarrow T X$ be a skew-adjoint operator such that

$$
\omega(u, v)=g^{T X}\left(J_{0} u, v\right), \quad u, v \in T X .
$$

Consider the operator $J: T X \rightarrow T X$ given by

$$
J=J_{0}\left(-J_{0}^{2}\right)^{-1 / 2} .
$$

Then $J$ is an almost complex structure compatible with $\omega$ and $g^{T X}$, that is, $g^{T X}(J u, J v)=$ $g^{T X}(u, v), \omega(J u, J v)=\omega(u, v)$ for any $u, v \in T X$ and $\omega(u, J u)>0$ for any $u \in T X, u \neq 0$.

Let $\nabla^{L^{p} \otimes E}: \mathscr{C}^{\infty}\left(X, L^{p} \otimes E\right) \rightarrow \mathscr{C}^{\infty}\left(X, T^{*} X \otimes L^{p} \otimes E\right)$ be the connection on $L^{p} \otimes E$ induced by $\nabla^{L}$ and $\nabla^{E}$. Denote by $\Delta^{L^{p} \otimes E}$ the induced Bochner-Laplacian acting on $\mathscr{C}^{\infty}\left(X, L^{p} \otimes E\right)$ by

$$
\Delta^{L^{p} \otimes E}=\left(\nabla^{L^{p} \otimes E}\right)^{*} \nabla^{L^{p} \otimes E}
$$

where $\left(\nabla^{L^{p} \otimes E}\right)^{*}: \mathscr{C}^{\infty}\left(X, T^{*} X \otimes L^{p} \otimes E\right) \rightarrow \mathscr{C}^{\infty}\left(X, L^{p} \otimes E\right)$ denotes the formal adjoint of the operator $\nabla^{L^{p} \otimes E}$. The renormalized Bochner-Laplacian is a differential operator $\Delta_{p}$ acting on $\mathscr{C}^{\infty}\left(X, L^{p} \otimes E\right)$ by

$$
\Delta_{p}=\Delta^{L^{p} \otimes E}-p \tau
$$

where $\tau \in \mathscr{C}^{\infty}(X)$ is given by

$$
\tau(x)=-\pi \operatorname{Tr}\left[J_{0}(x) J(x)\right], \quad x \in X .
$$

The renormalized Bochner-Laplacian was introduced by Guillemin and Uribe in [11]. When $(X, \omega)$ is a Kähler manifold, it is twice the corresponding Kodaira-Laplacian on functions $\square^{L^{p}}=\bar{\partial}^{L^{p} *} \bar{\partial}^{L^{p}}$. The asymptotic of the spectrum of the operator $\Delta_{p}$ as $p \rightarrow \infty$ was studied in $[3,[5,11,21,22]$.

In this article, we will suppose that $\left(X, g^{T X}\right)$ is complete and $R^{L}, R^{E}, J, g^{T X}$ have bounded geometry (i.e., they and their derivatives of any order are uniformly bounded on $X$ in the norm induced by $g^{T X}, h^{L}$ and $h^{E}$, and the injectivity radius of $\left(X, g^{T X}\right)$ is positive). We will also assume that

$$
\mu_{0}=\inf _{\substack{x \in X \\ u \in T_{x} X \backslash\{0\}}} \frac{i R_{x}^{L}(u, J(x) u)}{|u|_{g^{T X}}^{2}}>0 .
$$

Note that $\lambda(x)=\inf _{u \in T_{x} X \backslash\{0\}} i R_{x}^{L}(u, J(x) u) /|u|_{g^{T X}}^{2}$ is the smallest eigenvalue of $i R_{x}^{L}(\cdot, J(x) \cdot)$ with respect to $g_{x}^{T X}$, for $x \in X$. Thus (1.7) is a condition of uniform positivity of $R^{L}$ with respect to $g^{T X}$. 
Since $\left(X, g^{T X}\right)$ is complete, the Bochner-Laplacian and the renormalized Bochner-Laplacian $\Delta_{p}$ are essentially self-adjoint, see Theorem 2.4 . We still denote by $\Delta_{p}$ the unique self-adjoint extension of $\Delta_{p}: \mathscr{C}_{c}^{\infty}\left(X, L^{p} \otimes E\right) \rightarrow \mathscr{C}_{c}^{\infty}\left(X, L^{p} \otimes E\right)$ acting on compactly supported smooth sections, and by $\sigma\left(\Delta_{p}\right)$ its spectrum in $L^{2}\left(X, L^{p} \otimes E\right)$. First, we state the following spectral gap property for the operator $\Delta_{p}$ which is a direct consequence of [26, Lemma 1].

Theorem 1.1. Let $(X, \omega)$ be a symplectic manifold with a prequantum line bundle $\left(L, \nabla^{L}, h^{L}\right)$. Let $g^{T X}$ be a complete Riemannian metric on $X$ and let $J$ be the almost complex structure defined by (1.3). Let $\left(E, \nabla^{E}, h^{E}\right)$ be an auxiliary vector bundle on $X$. We assume that $R^{L}, R^{E}, J, g^{T X}$ have bounded geometry and (1.7) holds. Then there exists $C_{L}>0$ such that for any $p \in \mathbb{N}$ the spectrum of the renormalized Bochner-Laplacian (1.5) satisfies

$$
\sigma\left(\Delta_{p}\right) \subset\left[-C_{L}, C_{L}\right] \cup\left[2 p \mu_{0}-C_{L},+\infty\right) .
$$

When $X$ is compact and $E$ is the trivial line bundle, this theorem (with a not precised constant $\mu_{0}$ ) is the main result of Guillemin and Uribe [11]. For a general vector bundle $E$, it was proved by Ma and Marinescu [21, Corollary 1.2], cf. also [22, Theorem 8.3.1], with the geometric constant $\mu_{0}$ given by (1.7). The analogous theorem for the $\operatorname{spin}^{c}$ Dirac operator on a manifold of bounded geometry is stated in [26, Lemma 1]. Theorem 1.1 can be directly derived from this result, following the proof of [21, Corollary 1.2] (see also [21, Corollary 4.7] for the case of a covering of a compact manifold), thus we will not repeat this proof here. Note that there are cases when the renormalized Bochner-Laplacian has a spectral gap even if the curvature $R^{L}$ degenerates at finite order [27, Remark 22].

For a Borel set $B \subset \mathbb{R}$, we denote by $\mathcal{E}\left(B, \Delta_{p}\right)$ the spectral projection corresponding to the subset $B$. Consider the spectral space $\mathcal{H}_{p} \subset L^{2}\left(X, L^{p} \otimes E\right)$ of $\Delta_{p}$ corresponding to $\left[-C_{L}, C_{L}\right]$,

$$
\mathcal{H}_{p}:=\text { Range } \mathcal{E}\left(\left[-C_{L}, C_{L}\right], \Delta_{p}\right) \text {. }
$$

If $X$ is compact, the spectrum of $\Delta_{p}$ is discrete and $\mathcal{H}_{p}$ is the subspace spanned by the eigensections of $\Delta_{p}$ corresponding to eigenvalues in $\left[-C_{L}, C_{L}\right]$. Let

$$
P_{\mathcal{H}_{p}}:=\mathcal{E}\left(\left[-C_{L}, C_{L}\right], \Delta_{p}\right): L^{2}\left(X, L^{p} \otimes E\right) \longrightarrow \mathcal{H}_{p}
$$

be the orthogonal projection. Let $\pi_{1}$ and $\pi_{2}$ be the projections of $X \times X$ on the first and second factor. The Schwartz kernel of the operator $P_{\mathcal{H}_{p}}$ with respect to the Riemannian volume form $d v_{X}$ is a smooth section $P_{p}(\cdot, \cdot) \in \mathscr{C}^{\infty}\left(X \times X, \pi_{1}^{*}\left(L^{p} \otimes E\right) \otimes \pi_{2}^{*}\left(L^{p} \otimes E\right)^{*}\right)$, see [22, Remark 1.4.3]. It is called the generalized Bergman kernel of $\Delta_{p}$ in [23], since it generalizes the Bergman kernel on complex manifolds.

Theorem 1.2. Under the assumptions of Theorem 1.1 there exists $c>0$ such that for any $k \in \mathbb{N}$, there exists $C_{k}>0$ such that for any $p \in \mathbb{N}, x, x^{\prime} \in X$, we have

$$
\left|P_{p}\left(x, x^{\prime}\right)\right|_{\mathscr{C}^{k}} \leq C_{k} p^{n+\frac{k}{2}} e^{-c \sqrt{p} d\left(x, x^{\prime}\right)} .
$$

Here $d\left(x, x^{\prime}\right)$ is the geodesic distance and $\left|P_{p}\left(x, x^{\prime}\right)\right|_{\mathscr{C}^{k}}$ denotes the pointwise $\mathscr{C}^{k}$-seminorm of the section $P_{p}$ at a point $\left(x, x^{\prime}\right) \in X \times X$, which is the sum of the norms induced by $h^{L}, h^{E}$ and $g^{T X}$ of the derivatives up to order $k$ of $P_{p}$ with respect to the connection $\nabla^{L^{p} \otimes E}$ and the Levi-Civita connection $\nabla^{T X}$ evaluated at $\left(x, x^{\prime}\right)$.

For the Bergman kernel of the $\operatorname{spin}^{c}$ Dirac operator associated to a positive line bundle on a symplectic manifold of bounded geometry, the same type of exponential estimate is proved in 
[26, Theorem 1] (see also the references therein for the previous results). In [26], the authors use the methods of [10, 22, 23] based on the spectral gap property of the $\operatorname{spin}^{c}$ Dirac operator, finite propagation speed arguments for the wave equation, the heat semigroup and rescaling of the $\operatorname{spin}^{c}$ Dirac operator near the diagonal, which is inspired by the analytic localization technique of Bismut-Lebeau [2]. It is important in [26] that the eigenvalues of the associated Laplacian are either 0 or tend to $+\infty$. In the current situation, the renormalized BochnerLaplacian has possibly different bounded eigenvalues, which makes difficult to use the heat semigroup technique. So we replace the heat semigroup technique by a different approach, which was developed by the first author in [15]: We follow essentially the general strategy of [10, 22, 23] but use weighted estimates with appropriate exponential weights as in [14] instead of the use of the heat semigroup and finite propagation speed arguments. In [16], this approach is used to prove asymptotic decay of order $\mathcal{O}\left(e^{-c \sqrt{p}}\right)$ for eigenfunctions of a self-adjoint Toeplitz operator with discrete wells associated with the renormalized BochnerLaplacian in the classically forbidden region.

As an application of our proof of Theorem 1.2, we obtain the relation between the generalized Bergman kernel on a Galois covering of a compact symplectic manifold and the generalized Bergman kernel on the base as an analog of [26, Theorem 2] for the Bergman kernel of the $\operatorname{spin}^{c}$ Dirac operator.

Theorem 1.3. Let $(X, \omega)$ be a compact symplectic manifold. Let $\left(L, \nabla^{L}, h^{L}\right),\left(E, \nabla^{E}, h^{E}\right), g^{T X}$ be given as above. Consider a Galois covering $\pi: \widetilde{X} \rightarrow X$ and let $\Gamma$ be the group of deck transformations. Denote by $\widetilde{\omega},\left(\widetilde{L}, \nabla^{\widetilde{L}}, h^{\widetilde{L}}\right),\left(\widetilde{E}, \nabla^{\widetilde{E}}, h^{\widetilde{E}}\right), g^{T \widetilde{X}}$ be the lifts of the above data to $\widetilde{X}$. Let $\widetilde{\Delta}_{p}$ be the renormalized Bochner-Laplacian acting on $\mathscr{C}^{\infty}\left(\widetilde{X}, \widetilde{L}^{p} \otimes \widetilde{E}\right)$ and $\widetilde{P}_{p}(\cdot, \cdot)$ be the generalized Bergman kernel of $\widetilde{\Delta}_{p}$. There exists $p_{1} \in \mathbb{N}$ such that for any $p>p_{1}$ we have for any $x, x^{\prime} \in \tilde{X}$,

$$
\sum_{\gamma \in \Gamma} \widetilde{P}_{p}\left(\gamma x, x^{\prime}\right)=P_{p}\left(\pi(x), \pi\left(x^{\prime}\right)\right)
$$

This type of results has a long history. In the category of complex manifolds it appeared in connection with the theory of automorphic forms and Poincare series in the works of Selberg and Godement. Earle [9] gave a proof when $\widetilde{X}$ is a bounded symmetric domain (under some hypothesis on the variation of Bergman kernels). The second and third authors proved (1.12) for the Bergman kernels associated to the $\operatorname{spin}^{c}$ Dirac operator on a symplectic manifold, in particular, in the Kähler case [26, Theorem 2]. Lu and Zelditch [18] independently proved (1.12) for the Bergman kernels on Kähler manifolds when $E=\mathbb{C}$.

As another application of the technique developed in this article, we extend the results on the full off-diagonal asymptotic expansion of the generalized Bergman kernels of the renormalized Bochner-Laplacians associated to high tensor powers of a positive line bundle over a compact symplectic manifold, obtained in [17, 15], to the case of manifolds of bounded geometry and slightly improve the remainder estimate in the asymptotic expansions, proving an exponential estimate $\mathcal{O}\left(e^{-c_{0} \sqrt{p}}\right)$ instead of $\mathcal{O}\left(p^{-\infty}\right)$ (see Theorem 4.3 below).

Finally, we study the theory of Berezin-Toeplitz quantization on symplectic orbifolds by using as quantum spaces the spectral spaces $\mathcal{H}_{p}$, especially we show that the set of Toeplitz operators forms an algebra. Ma and Marinescu obtained first Berezin-Toeplitz quantization on symplectic orbifolds by using as quantum spaces the kernel of the $\operatorname{spin}^{c}$ Dirac operator, 
in particular, on compact complex orbifolds [24, Theorems 6.13, 6.16]. Let us note also that Hsiao and Marinescu [13] constructed a Berezin-Toeplitz quantization for eigenstates of small eigenvalues in the case of complex manifolds. For a comprehensive introduction to this subject see [20, 22, 25].

The article is organized as follows. In Section 2, we collect some necessary background information on differential operators and Sobolev spaces on manifolds of bounded geometry. In Section 3, we remind some results on weighted estimates on manifolds of bounded geometry and prove Theorems 1.2 and 1.3 . Section 4 is devoted to the full off-diagonal asymptotic expansions. In Section 5 we study Berezin-Toeplitz quantization on symplectic orbifolds.

\section{PRELIMINARIES ON DifFERENTIAL OPERATORS AND SOBOLEV SPACES}

In this section, we collect some necessary background information on differential operators and Sobolev spaces on manifolds of bounded geometry. We refer the reader to [14, 26] for more information. The novel point is that our constructions are adapted to a particular sequence of vector bundles $L^{p} \otimes E, p \in \mathbb{N}$. This concerns with a specific choice of the Sobolev norm as well as with a slightly refined form of the Sobolev embedding theorem. We will keep the setting described in Introduction.

2.1. Differential operators. Let $\mathcal{F}$ be a vector bundle over $X$. Suppose that $\mathcal{F}$ is Euclidean or Hermitian depending on whether it is real or complex and equipped with a metric connection $\nabla^{\mathcal{F}}$. The Levi-Civita connection $\nabla^{T X}$ on $\left(X, g^{T X}\right)$ and the connection $\nabla^{\mathcal{F}}$ define a metric connection $\nabla^{\mathcal{F}}: \mathscr{C}^{\infty}\left(X,\left(T^{*} X\right)^{\otimes j} \otimes \mathcal{F}\right) \rightarrow \mathscr{C}^{\infty}\left(X,\left(T^{*} X\right)^{\otimes(j+1)} \otimes \mathcal{F}\right)$ on each vector bundle $\left(T^{*} X\right)^{\otimes j} \otimes \mathcal{F}$ for $j \in \mathbb{N}$, that allows us to introduce the operator

$$
\left(\nabla^{\mathcal{F}}\right)^{\ell}: \mathscr{C}^{\infty}(X, \mathcal{F}) \rightarrow \mathscr{C}^{\infty}\left(X,\left(T^{*} X\right)^{\otimes \ell} \otimes \mathcal{F}\right)
$$

for every $\ell \in \mathbb{N}$. Any differential operator $A$ of order $q$ acting in $\mathscr{C}^{\infty}(X, \mathcal{F})$ can be written as

$$
A=\sum_{\ell=0}^{q} a_{\ell} \cdot\left(\nabla^{\mathcal{F}}\right)^{\ell}
$$

where $a_{\ell} \in \mathscr{C}^{\infty}\left(X,(T X)^{\otimes \ell}\right)$ and the endomorphism $\cdot:(T X)^{\otimes \ell} \otimes\left(\left(T^{*} X\right)^{\otimes \ell} \otimes \mathcal{F}\right) \rightarrow \mathcal{F}$ is given by the contraction.

If $\mathcal{F}$ has bounded geometry, we denote by $\mathscr{C}_{b}^{k}(X, \mathcal{F})$ the space of sections $u \in \mathscr{C}^{k}(X, \mathcal{F})$ such that

$$
\|u\|_{\mathscr{C}_{b}^{k}}=\sup _{x \in X, \ell \leq k}\left|\left(\nabla^{\mathcal{F}}\right)^{\ell} u(x)\right|<\infty
$$

where $|\cdot|_{x}$ is the norm in $\left(T_{x}^{*} X\right)^{\otimes \ell} \otimes \mathcal{F}_{x}$ defined by $g^{T X}$ and $h^{\mathcal{F}}$. We also denote by $B D^{q}(X, \mathcal{F})$ the space of differential operators $A$ of order $q$ in $\mathscr{C}_{c}^{\infty}(X, \mathcal{F})$ with coefficients $a_{\ell}$ in $\mathscr{C}_{b}^{\infty}\left(X,(T X)^{\otimes \ell}\right)$.

Usually, we will deal with families of differential operators of the form

$$
\left\{A_{p} \in B D^{q}\left(X, L^{p} \otimes E\right), p \in \mathbb{N}^{*}\right\} .
$$

We will say that such a family $\left\{A_{p} \in B D^{q}\left(X, L^{p} \otimes E\right), p \in \mathbb{N}^{*}\right\}$ is bounded in $p$, if

$$
A_{p}=\sum_{\ell=0}^{q} a_{p, \ell} \cdot\left(\frac{1}{\sqrt{p}} \nabla^{L^{p} \otimes E}\right)^{\ell}, \quad a_{p, \ell} \in \mathscr{C}_{b}^{\infty}\left(X,(T X)^{\otimes \ell}\right)
$$


and, for any $\ell=0,1, \ldots, q$, the family $\left\{a_{p, \ell}, p \in \mathbb{N}^{*}\right\}$ is bounded in the Frechet space $\mathscr{C}_{b}^{\infty}\left(X,(T X)^{\otimes \ell}\right)$. An example of a bounded in $p$ family of differential operators is given by $\left\{\frac{1}{p} \Delta_{p}: p \in \mathbb{N}^{*}\right\}$.

2.2. Sobolev spaces. Denote by $d v_{X}$ the Riemannian volume form of $\left(X, g^{T X}\right)$. The $L^{2}$-norm on $L^{2}\left(X, L^{p} \otimes E\right)$ is given by

$$
\|u\|_{p, 0}^{2}=\int_{X}|u(x)|^{2} d v_{X}(x), \quad u \in L^{2}\left(X, L^{p} \otimes E\right) .
$$

For any integer $m>0$, we introduce the norm $\|\cdot\|_{p, m}$ on $\mathscr{C}_{c}^{\infty}\left(X, L^{p} \otimes E\right)$ by the formula

$$
\|u\|_{p, m}^{2}=\sum_{\ell=0}^{m} \int_{X}\left|\left(\frac{1}{\sqrt{p}} \nabla^{L^{p} \otimes E}\right)^{\ell} u(x)\right|^{2} d v_{X}(x), \quad u \in H^{m}\left(X, L^{p} \otimes E\right) .
$$

The completion of $\mathscr{C}_{c}^{\infty}\left(X, L^{p} \otimes E\right)$ with respect to $\|\cdot\|_{p, m}$ is the Sobolev space $H^{m}\left(X, L^{p} \otimes E\right)$ of order $m$. Denote by $\langle\cdot, \cdot\rangle_{p, m}$ the corresponding inner product on $H^{m}\left(X, L^{p} \otimes E\right)$. For any integer $m<0$, we define the norm in the Sobolev space $H^{m}\left(X, L^{p} \otimes E\right)$ by duality. For any bounded linear operator $A: H^{m}\left(X, L^{p} \otimes E\right) \rightarrow H^{m^{\prime}}\left(X, L^{p} \otimes E\right), m, m^{\prime} \in \mathbb{Z}$, we will denote its operator norm by $\|A\|_{p}^{m, m^{\prime}}$.

One can easily derive the following mapping properties of differential operators in Sobolev spaces.

Proposition 2.1. Any operator $A \in B D^{q}\left(X, L^{p} \otimes E\right)$ defines a bounded operator

$$
A: H^{m+q}\left(X, L^{p} \otimes E\right) \longrightarrow H^{m}\left(X, L^{p} \otimes E\right)
$$

for any $m \in \mathbb{N}$. Moreover, if a family $\left\{A_{p} \in B D^{q}\left(X, L^{p} \otimes E\right), p \in \mathbb{N}\right\}$ is bounded in $p$, then for any $m \in \mathbb{N}$, there exists $C_{m}>0$ such that, for all $p \in \mathbb{N}$,

$$
\left\|A_{p} u\right\|_{p, m} \leq C_{m}\|u\|_{p, m+q}, \quad u \in H^{m+q}\left(X, L^{p} \otimes E\right) .
$$

2.3. Sobolev embedding theorem. We will need a refined form of the Sobolev embedding theorem adapted to the sequence $L^{p} \otimes E, p \in \mathbb{N}$.

Proposition 2.2 ([26], Lemma 2). For any $k, m \in \mathbb{N}$ with $m>k+n$, we have an embedding

$$
H^{m}\left(X, L^{p} \otimes E\right) \subset \mathscr{C}_{b}^{k}\left(X, L^{p} \otimes E\right) .
$$

Moreover, there exists $C_{m, k}>0$ such that, for any $p \in \mathbb{N}^{*}$ and $u \in H^{m}\left(X, L^{p} \otimes E\right)$,

$$
\|u\|_{\mathscr{C}_{b}^{k}} \leq C_{m, k} p^{(n+k) / 2}\|u\|_{p, m}
$$

For any $x \in X$ and $v \in\left(L^{p} \otimes E\right)_{x}$, we define the delta-section $\delta_{v} \in \mathscr{C}^{-\infty}\left(X, L^{p} \otimes E\right)$ as a linear functional on $\mathscr{C}_{c}^{\infty}\left(X, L^{p} \otimes E\right)$ given by

$$
\left\langle\delta_{v}, \varphi\right\rangle=\langle v, \varphi(x)\rangle_{h^{L^{p} \otimes E}}, \quad \varphi \in \mathscr{C}_{c}^{\infty}\left(X, L^{p} \otimes E\right) .
$$

Proposition 2.3. For any $m>n$ and $v \in L^{p} \otimes E, \delta_{v} \in H^{-m}\left(X, L^{p} \otimes E\right)$ with the following norm estimate

$$
\sup _{|v|=1} p^{-n / 2}\left\|\delta_{v}\right\|_{p,-m}<\infty
$$


Proof. By Proposition 2.2 and the definition of the Sobolev norm, we have

$$
\left\|\delta_{v}\right\|_{p,-m} \leq C \sup _{\phi \in H^{m}\left(X, L^{p} \otimes E\right)} \frac{\left\langle\delta_{v}, \phi\right\rangle}{\|\phi\|_{p, m}} \leq C p^{n / 2}|v| .
$$

2.4. The renormalized Bochner-Laplacian. Let us first note the following basic result.

Theorem 2.4. Let $(X, \omega)$ be a symplectic manifold with a prequantum line bundle $\left(L, \nabla^{L}, h^{L}\right)$. Let $g^{T X}$ be a complete Riemannian metric on $X$ and let $\left(E, \nabla^{E}, h^{E}\right)$ be an auxiliary vector bundle.

(i) The space $\mathscr{C}_{c}^{\infty}\left(X, L^{p} \otimes E\right)$ is dense in the graph norm of the maximal extension of $\nabla^{L^{p} \otimes E}$ and $\mathscr{C}_{c}^{\infty}\left(X, T^{*} X \otimes L^{p} \otimes E\right)$ is dense in the graph norm of the maximal extension of $\left(\nabla^{L^{p} \otimes E}\right)^{*}$.

(ii) The Hilbert space adjoint of the maximal extension of $\nabla^{L^{p} \otimes E}$ coincides with the maximal extension of $\left(\nabla^{L^{p} \otimes E}\right)^{*}$.

(iii) The Bochner-Laplacian $\Delta^{L^{p} \otimes E}=\left(\nabla^{L^{p} \otimes E}\right)^{*} \nabla^{L^{p} \otimes E}$ acting on $\mathscr{C}_{c}^{\infty}\left(X, L^{p} \otimes E\right)$ is essentially selfadjoint. In particular, the renormalized Bochner-Laplacian $\Delta_{p}$ acting on $\mathscr{C}_{c}^{\infty}\left(X, L^{p} \otimes E\right)$ is essentially selfadjoint.

Proof. Assertion (i) is a form of the Andreotti-Vesentini Lemma [22, Lemma 3.3.1]. The proof is obtained by replacing $\bar{\partial}^{E}$ in [22, Lemma 3.3.1] with $\nabla^{L^{p} \otimes E}$. Assertions (ii) and (iii) are obtained by adapting in the same way the proofs of [22, Corollary 3.3.3] and [22, Corollary 3.3.4], respectively (valid for $\left(\bar{\partial}^{E}\right)^{*}$ and the Kodaira-Laplacian $\square^{E}$ ).

Now we establish some additional properties of the family $\left\{\frac{1}{p} \Delta_{p}, p \in \mathbb{N}\right\}$ of differential operators, which is bounded in $p$.

Theorem 2.5. There exist $C_{2}, C_{3}>0$ such that for any $p \in \mathbb{N}^{*}, u, u^{\prime} \in \mathscr{C}_{c}^{\infty}\left(X, L^{p} \otimes E\right)$,

$$
\begin{aligned}
& \left\langle\frac{1}{p} \Delta_{p} u, u\right\rangle_{p, 0} \geq\|u\|_{p, 1}^{2}-C_{2}\|u\|_{p, 0}^{2}, \\
& \left|\left\langle\frac{1}{p} \Delta_{p} u, u^{\prime}\right\rangle_{p, 0}\right| \leq C_{3}\|u\|_{p, 1}\left\|u^{\prime}\right\|_{p, 1} .
\end{aligned}
$$

Proof. These estimates follow immediately from the identity

$$
\left\langle\frac{1}{p} \Delta_{p} u, u\right\rangle_{p, 0}=\left\|\frac{1}{\sqrt{p}} \nabla^{L^{p} \otimes E} u\right\|_{p, 0}^{2}-\langle\tau u, u\rangle_{p, 0} .
$$

Let $\delta$ be the counterclockwise oriented circle in $\mathbb{C}$ centered at 0 of radius $\mu_{0}$.

Theorem 2.6. There exists $p_{0} \in \mathbb{N}$ such that for any $\lambda \in \delta$ and $p \geq p_{0}$ the operator $\lambda-\frac{1}{p} \Delta_{p}$ is invertible in $L^{2}\left(X, L^{p} \otimes E\right)$, and there exists $C>0$ such that for all $\lambda \in \delta$ and $p \geq p_{0}$ we have

$$
\left\|\left(\lambda-\frac{1}{p} \Delta_{p}\right)^{-1}\right\|_{p}^{0,0} \leq C, \quad\left\|\left(\lambda-\frac{1}{p} \Delta_{p}\right)^{-1}\right\|_{p}^{-1,1} \leq C .
$$

Proof. We will closely follow the proof of [10, Theorem 4.8] or [23, Theorem 1.7] (cf. also the proof of [2, Theorem 11.27]). The first estimate follows from Theorem 1.1] and the spectral theorem. By (2.12), we have, for $\lambda_{0} \leq-C_{2}$,

$$
\left\langle\left(\frac{1}{p} \Delta_{p}-\lambda_{0}\right) u, u\right\rangle_{p, 0} \geq\|u\|_{p, 1}^{2}
$$


therefore, the resolvent $\left(\lambda_{0}-\frac{1}{p} \Delta_{p}\right)^{-1}$ exists and

$$
\left\|\left(\lambda_{0}-\frac{1}{p} \Delta_{p}\right)^{-1}\right\|_{p}^{-1,1} \leq 1
$$

Now we can write, for $\lambda \in \delta$ and $\lambda_{0} \leq-C_{2}$,

$$
\left(\lambda-\frac{1}{p} \Delta_{p}\right)^{-1}=\left(\lambda_{0}-\frac{1}{p} \Delta_{p}\right)^{-1}-\left(\lambda-\lambda_{0}\right)\left(\lambda-\frac{1}{p} \Delta_{p}\right)^{-1}\left(\lambda_{0}-\frac{1}{p} \Delta_{p}\right)^{-1} .
$$

Thus for $\lambda \in \delta$, we get from the first estimate of (2.15), (2.17) and (2.18),

$$
\left\|\left(\lambda-\frac{1}{p} \Delta_{p}\right)^{-1}\right\|_{p}^{-1,0} \leq 1+C\left|\lambda-\lambda_{0}\right| \text {. }
$$

Changing the last two factors in (2.18) and applying (2.19), we get

$$
\left\|\left(\lambda-\frac{1}{p} \Delta_{p}\right)^{-1}\right\|_{p}^{-1,1} \leq 1+\left|\lambda-\lambda_{0}\right|\left(1+C\left|\lambda-\lambda_{0}\right|\right) .
$$

The proof of Theorem 2.6 is completed.

\section{Proof of MAIN RESUltS}

This section is devoted to the proofs of Theorems 1.2 and 1.3. First, we describe a class of exponential weight functions as in [14]. Then we prove norm estimates in weighted Sobolev spaces for the resolvent $\left(\lambda-\frac{1}{p} \Delta_{p}\right)^{-m}$. Here we follow general constructions of [10, 22, 23], which are inspired by the analytic localization technique of Bismut-Lebeau [2, $\S 11]$. Next, we derive pointwise exponential estimates for the Schwartz kernel of the operator $\left(\lambda-\frac{1}{p} \Delta_{p}\right)^{-m}$ and its derivatives of an arbitrary order, using a refined form of the Sobolev embedding theorem stated in Proposition 2.2. Finally, we use the formula as in [23, (1.55)]

$$
P_{\mathcal{H}_{p}}=\frac{1}{2 \pi i} \int_{\delta} \lambda^{m-1}\left(\lambda-\frac{1}{p} \Delta_{p}\right)^{-m} d \lambda, \quad m \geq 1,
$$

that allows us to complete the proofs of Theorems 1.2 and 1.3 .

3.1. Weight functions. Recall that $d$ denotes the distance function on $X$. By [14, Proposition 4,1], there exists a "smoothed distance" function $\widetilde{d} \in \mathscr{C}^{\infty}(X \times X)$, satisfying the following conditions:

(1) there is $r>0$ such that

$$
|\widetilde{d}(x, y)-d(x, y)|<r, \quad x, y \in X
$$

(2) for any $k>0$, there exists $C_{k}>0$ such that, for any multi-index $\beta$ with $|\beta|=k$,

$$
\left|\partial_{x}^{\beta} \widetilde{d}(x, y)\right|<C_{k}, \quad x, y \in X
$$

where the derivatives are taken with respect to normal coordinates defined by the exponential map at $x$.

Actually, we will work with a sequence of smoothed distance functions $\widetilde{d}_{p}, p \in \mathbb{N}$, to remove small distances effects of smoothing. As one can easily see from the proof of [14, Proposition 4.1], for any $\gamma \in(0,1]$, there exists a function $\widetilde{d} \in \mathscr{C}^{\infty}(X \times X)$, satisfying (3.2) with $r=\gamma$ and (3.3) with $C_{k}=c_{k} \gamma^{1-k}, c_{k}>0$ is independent of $\gamma$. Let us briefly describe its construction. 
Let $a^{X}$ be the injectivity radius of $\left(X, g^{T X}\right)$. We denote by $B^{X}(x, r)$ and $B^{T_{x} X}(0, r)$ the open balls in $X$ and $T_{x} X$ with center $x$ and radius $r$, respectively. For any $x_{0} \in X$, we identify $B^{T_{x_{0}} X}\left(0, a^{X}\right)$ with $B^{X}\left(x_{0}, a^{X}\right)$ via the exponential map $\exp _{x_{0}}^{X}: T_{x_{0}} X \rightarrow X$. One can show that, for $\varepsilon \in\left(0, a^{X}\right)$, the geodesic distance on $B^{X}\left(x_{0}, \varepsilon\right)$ is equivalent to the Euclidean distance on $B^{T_{x_{0}} X}(0, \varepsilon)$ uniformly on $x_{0} \in X$ : there exists $C>0$ such that for any $x_{0} \in X$ and $Z, W \in B^{T_{x_{0}} X}(0, \varepsilon)$,

$$
C^{-1} d^{T_{x_{0}} X}(Z, W) \leq d\left(\exp _{x_{0}}^{X}(Z), \exp _{x_{0}}^{X}(W)\right) \leq C d^{T_{x_{0}} X}(Z, W) .
$$

By [14, Lemma 2.3], for $\varepsilon<a^{X} / 2$, there exists a covering $\left\{B^{X}\left(x_{j}, \varepsilon\right)\right\}_{j \in \mathbb{N}}$ of $X$ and $N \in \mathbb{N}$ such that every intersection of $N+1$ balls $B^{X}\left(x_{j}, 2 \varepsilon\right)$ is empty. Furthermore, by [14, Lemma 2.4], there exists a partition of unity $\sum_{j=1}^{\infty} \phi_{j}=1$ subordinated to this covering such that supp $\phi_{j} \subset B^{X}\left(x_{j}, \varepsilon\right)$ for any $j$ and, for any $k \in \mathbb{N}$, there exists $C_{k}$ such that $\left|\partial^{\alpha} \phi_{j}(x)\right|<C_{k}$ for any $j, x \in B^{X}\left(x_{j}, \varepsilon\right)$ and $|\alpha|<k$, where the derivatives are computed with respect to the normal coordinates on $B^{X}\left(x_{j}, \varepsilon\right)$. Choose a function $\theta \in \mathscr{C}_{c}^{\infty}\left(\mathbb{R}^{2 n}\right)$ such that $\theta(x)=0$ if $|x|>1, \theta(x) \geq 0$ for any $x \in \mathbb{R}^{2 n}$ and $\int_{\mathbb{R}^{2 n}} \theta(x) d x=1$. For any $\delta>0$, put

$$
\theta_{\delta}(x)=\delta^{-2 n} \theta\left(\delta^{-1} x\right), \quad x \in \mathbb{R}^{2 n} .
$$

The function $\widetilde{d}$ is defined by

$$
\widetilde{d}(x, y)=\sum_{j=1}^{\infty} \phi_{j}(x) \int_{\mathbb{R}^{2 n}} \theta_{\delta}\left(\left(\exp _{x_{j}}^{X}\right)^{-1}(x)-z\right) d\left(\exp _{x_{j}}^{X}(z), y\right) d z .
$$

Using the formula

$$
d(x, y)=\sum_{j=1}^{\infty} \phi_{j}(x) \int_{\mathbb{R}^{2 n}} \theta_{\delta}\left(\left(\exp _{x_{j}}^{X}\right)^{-1}(x)-z\right) d(x, y) d z,
$$

the triangle inequality, the fact that $\operatorname{supp} \theta_{\delta} \subset B(0, \delta)$ and (3.4), we get

$$
|\widetilde{d}(x, y)-d(x, y)| \leq \sum_{j=1}^{\infty} \phi_{j}(x) \int_{\mathbb{R}^{2 n}} \theta_{\delta}\left(\left(\exp _{x_{j}}^{X}\right)^{-1}(x)-z\right) d\left(x, \exp _{x_{j}}^{X}(z)\right) d z \leq C \delta .
$$

Choosing $\delta<C^{-1} \gamma$, we obtain (3.2) with $r=\gamma$.

Differentiating (3.6) with respect to $x$, for any multi-index $\beta$ with $|\beta|=k$, we get

$$
\partial_{x}^{\beta} \widetilde{d}(x, y)=\sum_{\tau \leq \beta} C_{\beta \tau} \sum_{j=1}^{\infty} \partial_{x}^{\tau} \phi_{j}(x) \int_{\mathbb{R}^{2 n}} \partial_{x}^{\beta-\tau}\left[\theta_{\delta}\left(\left(\exp _{x_{j}}^{X}\right)^{-1}(x)-z\right)\right] d\left(\exp _{x_{j}}^{X}(z), y\right) d z
$$

with some constants $C_{\beta \tau}>0$. Taking into account that $\sum_{j=1}^{\infty} \phi_{j}=1$ and $\int_{\mathbb{R}^{2 n}} \theta_{\delta}(x) d x=1$, it is easy to see that, for $k>0$,

$$
\sum_{\tau \leq \beta} C_{\beta \tau} \sum_{j=1}^{\infty} \partial_{x}^{\tau} \phi_{j}(x) \int_{\mathbb{R}^{2 n}} \partial_{x}^{\beta-\tau}\left[\theta_{\delta}\left(\left(\exp _{x_{j}}^{X}\right)^{-1}(x)-z\right)\right] d z=0 .
$$

As above, using these formulas, the triangle inequality, the fact that $\operatorname{supp} \theta_{\delta} \subset B(0, \delta)$ and (3.4), for any multi-index $\beta$ with $|\beta|=k>0$, we infer

$$
\left|\partial_{x}^{\beta} \widetilde{d}(x, y)\right|<\sum_{\tau \leq \beta} C_{\beta \tau} \sum_{j=1}^{\infty}\left|\partial_{x}^{\tau} \phi_{j}(x)\right| \int_{\mathbb{R}^{2 n}}\left|\partial_{x}^{\beta-\tau}\left[\theta_{\delta}\left(\left(\exp _{x_{j}}^{X}\right)^{-1}(x)-z\right)\right]\right| d\left(x, \exp _{x_{j}}^{X}(z)\right) d z \leq C_{\beta} \delta^{1-k}
$$


For $\delta$ chosen as above, this gives (3.3) with $C_{k}=c_{k} \gamma^{1-k}, c_{k}>0$ is independent of $\gamma$.

We will use such a function $\widetilde{d}$ for $\gamma=\frac{1}{\sqrt{p}}, p \in \mathbb{N}^{*}$, denoting it by $\widetilde{d}_{p}$. So it satisfies the conditions:

(1) we have

$$
\left|\widetilde{d}_{p}(x, y)-d(x, y)\right|<\frac{1}{\sqrt{p}}, \quad \text { for any } x, y \in X
$$

(2) for any $k>0$, there exists $c_{k}>0$ such that, for any multi-index $\beta$ with $|\beta|=k$,

$$
\left|\left(\frac{1}{\sqrt{p}}\right)^{k} \partial_{x}^{\beta} \widetilde{d}_{p}(x, y)\right|<\frac{c_{k}}{\sqrt{p}}, \quad \text { for any } x, y \in X .
$$

For any $\alpha \in \mathbb{R}, p \in \mathbb{N}^{*}$ and $y \in X$, we introduce a weight function $f_{\alpha, p, y} \in \mathscr{C}^{\infty}(X)$ by

$$
f_{\alpha, p, y}(x)=e^{\alpha \widetilde{d}_{p, y}(x)}, \quad \text { for } x \in X,
$$

where $\widetilde{d}_{p, y}$ is a smooth function on $X$ given by

$$
\widetilde{d}_{p, y}(x)=\tilde{d}_{p}(x, y), \quad \text { for } x \in X .
$$

We don't introduce explicitly the weighted Sobolev spaces associated with $f_{\alpha, p, y}$. Instead, we will work with the operator families for $p \in \mathbb{N}^{*}, \alpha \in \mathbb{R}, y \in X$,

$$
A_{p ; \alpha, y}=f_{\alpha, p, y} A_{p} f_{\alpha, p, y}^{-1}: \mathscr{C}_{c}^{\infty}\left(X, L^{p} \otimes E\right) \rightarrow \mathscr{C}^{-\infty}\left(X, L^{p} \otimes E\right)
$$

defined by an operator family $\left\{A_{p}: \mathscr{C}_{c}^{\infty}\left(X, L^{p} \otimes E\right) \rightarrow \mathscr{C}^{-\infty}\left(X, L^{p} \otimes E\right), p \in \mathbb{N}^{*}\right\}$. In particular, the desired exponential estimate of the Bergman kernel will be derived from the fact that the operator $f_{\alpha, p, y} P_{\mathcal{H}_{p}} f_{\alpha, p, y}^{-1}$ is a smoothing operator in the scale of Sobolev spaces.

3.2. Weighted estimates for the renormalized Bochner-Laplacian. Observe that, for $v \in$ $\mathscr{C}^{\infty}(X, T X)$,

$$
\nabla_{\alpha, y ; v}^{L^{p} \otimes E}:=f_{\alpha, p, y} \nabla_{v}^{L^{p} \otimes E} f_{\alpha, p, y}^{-1}=\nabla_{v}^{L^{p} \otimes E}-\alpha v\left(\widetilde{d}_{p, y}\right) .
$$

Therefore, $\nabla_{\alpha, y ; v}^{L^{p} \otimes E} \in B D^{1}\left(X, L^{p} \otimes E\right)$. Moreover, for any $a>0$ and $v \in \mathscr{C}_{b}^{\infty}(X, T X)$, the family

$$
\left\{\frac{1}{\sqrt{p}} \nabla_{\alpha, y ; v}^{L^{p} \otimes E}: y \in X,|\alpha|<a \sqrt{p}\right\}
$$

is a family of operators from $B D^{1}\left(X, L^{p} \otimes E\right)$, uniformly bounded in $p$.

This immediately implies that, if $Q \in B D^{q}\left(X, L^{p} \otimes E\right)$, then, for any $\alpha \in \mathbb{R}$ and $y \in X$, the operator $f_{\alpha, p, y} Q f_{\alpha, p, y}^{-1}$ is in $B D^{q}\left(X, L^{p} \otimes E\right)$. Moreover, for any $a>0$, the family $\left\{f_{\alpha, p, y} Q f_{\alpha, p, y}^{-1}\right.$ : $y \in X,|\alpha|<a \sqrt{p}\}$ is a family of operators from $B D^{q}\left(X, L^{p} \otimes E\right)$, uniformly bounded in $p$.

Now the operator $\Delta_{p ; \alpha, y}:=f_{\alpha, p, y} \Delta_{p} f_{\alpha, p, y}^{-1}$ has the form

$$
\Delta_{p ; \alpha, y}=\Delta_{p}+\alpha A_{p ; y}+\alpha^{2} B_{p ; y},
$$

where $A_{p ; y} \in B D^{1}\left(X, L^{p} \otimes E\right)$ and $B_{p ; y} \in B D^{0}\left(X, L^{p} \otimes E\right)$. Moreover, for any $a>0$, the families $\left\{\frac{1}{\sqrt{p}} A_{p ; y}: p \in \mathbb{N}^{*}, y \in X\right\}$ and $\left\{B_{p ; y}: p \in \mathbb{N}^{*}, y \in X\right\}$ are uniformly bounded in $p$.

If $\left\{e_{j}, j=1, \ldots, 2 n\right\}$ is a local frame in $T X$ on a domain $U \subset X$, and functions $\Gamma_{j k}^{i} \in$ $\mathscr{C}^{\infty}(U), i, j, k=1, \ldots, 2 n$, are defined by $\nabla_{e_{j}}^{T X} e_{k}=\sum_{i} \Gamma_{j k}^{i} e_{i}$, then we have

$$
\Delta_{p}=-g^{j k}(Z)\left[\nabla_{e_{j}}^{L^{p} \otimes E} \nabla_{e_{k}}^{L^{p} \otimes E}-\Gamma_{j k}^{\ell}(Z) \nabla_{e_{\ell}}^{L^{p} \otimes E}\right]-p \tau(Z),
$$


where $\left(g^{j k}(Z)\right)_{j, k}$ is the inverse of the matrix $\left(\left\langle e_{i}, e_{j}\right\rangle(Z)\right)_{i, j}$ and

$$
\Delta_{p ; \alpha, y}=-g^{j k}(Z)\left[\nabla_{\alpha, y ; e_{j}}^{L^{p} \otimes E} \nabla_{\alpha, y ; e_{k}}^{L^{p} \otimes E}-\Gamma_{j k}^{\ell}(Z) \nabla_{\alpha, y ; e_{\ell}}^{L^{p} \otimes E}\right]-p \tau(Z) .
$$

In particular,

$$
\operatorname{Re} \Delta_{p ; \alpha, y}=\Delta_{p}-\alpha^{2} \sum_{j, k=1}^{2 n} g^{j k}(Z) e_{j}\left(\widetilde{d}_{p, y}\right) e_{k}\left(\widetilde{d}_{p, y}\right)=\Delta_{p}-\alpha^{2}\left|\nabla \widetilde{d}_{p, y}\right|_{g(Z)}^{2} .
$$

From (3.17), we easily get

$$
\begin{aligned}
& A_{p ; y}=-\sum_{j, k=1}^{2 n} g^{j k}(Z)\left(2 e_{j}\left(\widetilde{d}_{p, y}\right) \nabla_{e_{k}}^{L^{p} \otimes E}+e_{j}\left(e_{k}\left(\widetilde{d}_{p, y}\right)\right)-\Gamma_{j k}^{\ell}(Z) e_{\ell}\left(\widetilde{d}_{p, y}\right)\right), \\
& B_{p ; y}=-\sum_{j, k=1}^{2 n} g^{j k}(Z) e_{j}\left(\widetilde{d}_{p, y}\right) e_{k}\left(\widetilde{d}_{p, y}\right) .
\end{aligned}
$$

By Proposition 2.1, for any $m \in \mathbb{N}$, there exists $C_{m}>0$ such that, for any $p \in \mathbb{N}^{*}, y \in X$ and $u \in H^{m}\left(X, L^{p} \otimes E\right)$,

$$
\left\|A_{p ; y} u\right\|_{p, m-1} \leq C_{m} p^{1 / 2}\|u\|_{p, m}, \quad\left\|B_{p ; y} u\right\|_{p, m} \leq C_{m}\|u\|_{p, m} .
$$

We have the following extension of Theorem 2.5.

Theorem 3.1. There exist $C_{0}, C_{2}, C_{3}>0$ such that for any $p \in \mathbb{N}^{*}, \alpha \in \mathbb{R}, y \in X$ and $u, u^{\prime} \in \mathscr{C}_{c}^{\infty}\left(X, L^{p} \otimes E\right)$,

$$
\begin{aligned}
& \operatorname{Re}\left\langle\frac{1}{p} \Delta_{p ; \alpha, y} u, u\right\rangle_{p, 0} \geq\|u\|_{p, 1}^{2}-\left(C_{2}+C_{0} \frac{\alpha^{2}}{p}\right)\|u\|_{p, 0}^{2}, \\
& \left|\left\langle\frac{1}{p} \Delta_{p ; \alpha, y} u, u^{\prime}\right\rangle_{p, 0}\right| \leq C_{3}\left(\|u\|_{p, 1}\left\|u^{\prime}\right\|_{p, 1}+\left(\frac{|\alpha|}{\sqrt{p}}\|u\|_{p, 1}+\frac{\alpha^{2}}{p}\|u\|_{p, 0}\right)\left\|u^{\prime}\right\|_{p, 0}\right) .
\end{aligned}
$$

Proof. Using Theorem 2.5, (3.15), (3.18), and (3.20), we get

$$
\begin{aligned}
\operatorname{Re}\left\langle\frac{1}{p} \Delta_{p ; \alpha, y} u, u\right\rangle_{p, 0} & \geq\left\langle\frac{1}{p} \Delta_{p} u, u\right\rangle_{p, 0}-C_{0} \frac{\alpha^{2}}{p}\|u\|_{p, 0}^{2} \\
& \geq\|u\|_{p, 1}^{2}-\left(C_{2}+C_{0} \frac{\alpha^{2}}{p}\right)\|u\|_{p, 0}^{2},
\end{aligned}
$$

and

$$
\begin{aligned}
& \left|\left\langle\frac{1}{p} \Delta_{p ; \alpha, y} u, u^{\prime}\right\rangle_{p, 0}\right| \\
& \leq\left|\left\langle\frac{1}{p} \Delta_{p} u, u^{\prime}\right\rangle_{p, 0}\right|+|\alpha|\left|\left\langle\frac{1}{p} A_{p, y} u, u^{\prime}\right\rangle_{p, 0}\right|+\alpha^{2}\left|\left\langle\frac{1}{p} B_{p, y} u, u^{\prime}\right\rangle_{p, 0}\right| \\
& \leq C_{3}\left(\|u\|_{p, 1}\left\|u^{\prime}\right\|_{p, 1}+\frac{|\alpha|}{\sqrt{p}}\|u\|_{p, 1}\left\|u^{\prime}\right\|_{p, 0}+\frac{\alpha^{2}}{p}\|u\|_{p, 0}\left\|u^{\prime}\right\|_{p, 0}\right) .
\end{aligned}
$$


3.3. Weighted estimates for the resolvent. Theorems 3.2-3.4 are the weighted analogs of [10, Theorems 4.8-4.10], [23, Theorems 1.7-1.9] which are inspired by [2, $\S 11]$. Now we extend Theorem 2.6 to the setting of weighted spaces.

Theorem 3.2. There exist $c>0, C>0$ and $p_{0} \in \mathbb{N}$ such that, for all $\lambda \in \delta, p \geq p_{0},|\alpha|<c \sqrt{p}$, $y \in X$, the operator $\lambda-\frac{1}{p} \Delta_{p ; \alpha, y}$ is invertible in $L^{2}\left(X, L^{p} \otimes E\right)$, and we have

$$
\left\|\left(\lambda-\frac{1}{p} \Delta_{p ; \alpha, y}\right)^{-1}\right\|_{p}^{0,0} \leq C, \quad\left\|\left(\lambda-\frac{1}{p} \Delta_{p ; \alpha, y}\right)^{-1}\right\|_{p}^{-1,1} \leq C .
$$

Proof. Let us denote in this proof

$$
R\left(\lambda, \frac{1}{p} \Delta_{p}\right):=\left(\lambda-\frac{1}{p} \Delta_{p}\right)^{-1}, \quad R\left(\lambda, \frac{1}{p} \Delta_{p ; \alpha, y}\right):=\left(\lambda-\frac{1}{p} \Delta_{p ; \alpha, y}\right)^{-1} .
$$

By Theorem 2.6, (3.15) and (3.20), it follows that, for all $\lambda \in \delta, p \in \mathbb{N}^{*}, \alpha \in \mathbb{R}$ and $y \in X$, we have

$$
\begin{aligned}
& \left\|\left(\Delta_{p ; \alpha, y}-\Delta_{p}\right) R\left(\lambda, \frac{1}{p} \Delta_{p}\right)\right\|_{p}^{-1,0}=\left\|\left(\alpha A_{p, y}+\alpha^{2} B_{p, y}\right) R\left(\lambda, \frac{1}{p} \Delta_{p}\right)\right\|_{p}^{-1,0} \\
& \leq C\left(|\alpha| \sqrt{p}\left\|R\left(\lambda, \frac{1}{p} \Delta_{p}\right)\right\|_{p}^{-1,1}+\alpha^{2}\left\|R\left(\lambda, \frac{1}{p} \Delta_{p}\right)\right\|_{p}^{-1,0}\right) \leq C\left(|\alpha| \sqrt{p}+\alpha^{2}\right) .
\end{aligned}
$$

Choose $c>0$ such that $C\left(c+c^{2}\right)<\frac{1}{2}$. Then, if $|\alpha|<c \sqrt{p}$, we have

$$
\left\|\left(\frac{1}{p} \Delta_{p ; \alpha, y}-\frac{1}{p} \Delta_{p}\right) R\left(\lambda, \frac{1}{p} \Delta_{p}\right)\right\|_{p}^{0,0} \leq\left\|\left(\frac{1}{p} \Delta_{p ; \alpha, y}-\frac{1}{p} \Delta_{p}\right) R\left(\lambda, \frac{1}{p} \Delta_{p}\right)\right\|_{p}^{-1,0}<\frac{1}{2} .
$$

Therefore, for all $\lambda \in \delta, p \in \mathbb{N}^{*}, \alpha \in \mathbb{R},|\alpha|<c \sqrt{p}$, and $y \in X$, the operator $\lambda-\frac{1}{p} \Delta_{p ; \alpha, y}$ is invertible in $L^{2}$, and we have

$$
R\left(\lambda, \frac{1}{p} \Delta_{p ; \alpha, y}\right)=R\left(\lambda, \frac{1}{p} \Delta_{p}\right)+R\left(\lambda, \frac{1}{p} \Delta_{p}\right) \sum_{j=1}^{\infty}\left(\left(\frac{1}{p} \Delta_{p ; \alpha, y}-\frac{1}{p} \Delta_{p}\right) R\left(\lambda, \frac{1}{p} \Delta_{p ; \alpha, y}\right)\right)^{j} .
$$

Therefore, by (2.15), (3.26) and (3.27), we get

$$
\begin{aligned}
& \left\|R\left(\lambda, \frac{1}{p} \Delta_{p ; \alpha, y}\right)\right\|_{p}^{-1,1} \leq\left\|R\left(\lambda, \frac{1}{p} \Delta_{p}\right)\right\|_{p}^{-1,1} \\
& +\left\|R\left(\lambda, \frac{1}{p} \Delta_{p}\right)\right\|_{p}^{0,1} \sum_{j=1}^{\infty}\left\|\left(\left(\frac{1}{p} \Delta_{p ; \alpha, y}-\frac{1}{p} \Delta_{p}\right) R\left(\lambda, \frac{1}{p} \Delta_{p ; \alpha, y}\right)\right)^{j-1}\right\|_{p}^{0,0} \\
& \times\left\|\left(\frac{1}{p} \Delta_{p ; \alpha, y}-\frac{1}{p} \Delta_{p}\right) R\left(\lambda, \frac{1}{p} \Delta_{p ; \alpha, y}\right)\right\|_{p}^{-1,0} \\
& \leq C+C \sum_{j=1}^{\infty} 2^{-j}=2 C .
\end{aligned}
$$

Since $\|\cdot\|_{p}^{0,0} \leq\|\cdot\|_{p}^{-1,1}$, (3.28) entails (3.24).

In the sequel, we will keep notation $c$ for the constant given by Theorem 3.2, which will be usually related with the interval $(-c \sqrt{p}, c \sqrt{p})$ of admissible values of the parameter $\alpha$. 
Remark 3.3. Observe that, for any $\lambda \in \delta, p \geq p_{0}, \alpha \in \mathbb{R}$ and $y \in X$, the operators $(\lambda-$ $\left.\frac{1}{p} \Delta_{p ; \alpha, y}\right)^{-1}$ and $\left(\lambda-\frac{1}{p} \Delta_{p}\right)^{-1}$ are related by the identity

$$
\left(\lambda-\frac{1}{p} \Delta_{p ; \alpha, y}\right)^{-1}=f_{\alpha, p, y}\left(\lambda-\frac{1}{p} \Delta_{p}\right)^{-1} f_{\alpha, p, y}^{-1},
$$

which should be understood in the following way. If $\alpha<0$, then, for any $s \in \mathscr{C}_{c}^{\infty}\left(X, L^{p} \otimes E\right)$, the expression $f_{\alpha, p, y}\left(\lambda-\frac{1}{p} \Delta_{p}\right)^{-1} f_{\alpha, p, y}^{-1} s$ makes sense and defines a function in $L^{2}\left(X, L^{p} \otimes E\right)$. Thus, we get a well-defined operator

$$
f_{\alpha, p, y}\left(\lambda-\frac{1}{p} \Delta_{p}\right)^{-1} f_{\alpha, p, y}^{-1}: \mathscr{C}_{c}^{\infty}\left(X, L^{p} \otimes E\right) \rightarrow L^{2}\left(X, L^{p} \otimes E\right),
$$

and one can check that $f_{\alpha, p, y}\left(\lambda-\frac{1}{p} \Delta_{p}\right)^{-1} f_{\alpha, p, y}^{-1} u=\left(\lambda-\frac{1}{p} \Delta_{p ; \alpha, y}\right)^{-1} u$ for any $u \in \mathscr{C}_{c}^{\infty}\left(X, L^{p} \otimes E\right)$. So (3.29) means that the operator $f_{\alpha, p, y}\left(\lambda-\frac{1}{p} \Delta_{p}\right)^{-1} f_{\alpha, p, y}^{-1}$ extends to a bounded operator in $L^{2}\left(X, L^{p} \otimes E\right)$, which coincides with $\left(\lambda-\frac{1}{p} \Delta_{p ; \alpha, y}\right)^{-1}$. If $\alpha>0$, then, for any $u \in L^{2}\left(X, L^{p} \otimes E\right)$, the expression $f_{\alpha, p, y}\left(\lambda-\frac{1}{p} \Delta_{p}\right)^{-1} f_{\alpha, p, y}^{-1} u$ makes sense as a distribution on $X$. Thus, we get a well-defined operator

$$
f_{\alpha, p, y}\left(\lambda-\frac{1}{p} \Delta_{p}\right)^{-1} f_{\alpha, p, y}^{-1}: L^{2}\left(X, L^{p} \otimes E\right) \rightarrow \mathscr{C}^{-\infty}\left(X, L^{p} \otimes E\right) .
$$

So (3.29) means that this operator is indeed a bounded operator in $L^{2}\left(X, L^{p} \otimes E\right)$, which coincides with $\left(\lambda-\frac{1}{p} \Delta_{p ; \alpha, y}\right)^{-1}$.

Theorem 3.4. For any $p \in \mathbb{N}^{*}, p \geq p_{0}, \lambda \in \delta, m \in \mathbb{N}, y \in X$ and $|\alpha|<c \sqrt{p}$ with the constant $c$ as in Theorem 3.2, the resolvent $\left(\lambda-\frac{1}{p} \Delta_{p ; \alpha, y}\right)^{-1}$ maps $H^{m}\left(X, L^{p} \otimes E\right)$ to $H^{m+1}\left(X, L^{p} \otimes E\right)$. Moreover, for any $m \in \mathbb{N}$, there exists $C_{m}>0$ such that for any $p \geq p_{0}, \lambda \in \delta, y \in X$ and $|\alpha|<c \sqrt{p}$

$$
\left\|\left(\lambda-\frac{1}{p} \Delta_{p ; \alpha, y}\right)^{-1}\right\|_{p}^{m, m+1} \leq C_{m} .
$$

Proof. The first statement of the theorem is a consequence of a general fact about operators on manifolds of bounded geometry. The operator $\left(\lambda-\frac{1}{p} \Delta_{p ; \alpha, y}\right)^{-1}$ is a pseudodifferential operator of order -2 , so it maps $H^{m}\left(X, L^{p} \otimes E\right)$ to $H^{m+2}\left(X, L^{p} \otimes E\right)$. It remains to prove the norm estimate (3.32).

To prove (3.32), first, we introduce normal coordinates near an arbitrary point $x_{0} \in X$. As above, we will identify the balls $B^{T_{x_{0}} X}\left(0, a^{X}\right)$ and $B^{X}\left(x_{0}, a^{X}\right)$ via the exponential map $\exp _{x_{0}}^{X}: T_{x_{0}} X \rightarrow X$. Furthermore, we choose a trivialization of the bundle $L$ and $E$ over $B^{X}\left(x_{0}, a^{X}\right)$, identifying the fibers $L_{Z}$ and $E_{Z}$ of $L$ and $E$ at $Z \in B^{T_{x_{0}} X}\left(0, a^{X}\right) \cong B^{X}\left(x_{0}, a^{X}\right)$ with $L_{x_{0}}$ and $E_{x_{0}}$ by parallel transport with respect to the connection $\nabla^{L}$ and $\nabla^{E}$ along the curve $\gamma_{Z}:[0,1] \ni u \rightarrow \exp _{x_{0}}^{X}(u Z)$. Denote by $\nabla^{L^{p} \otimes E}$ and $h^{L^{p} \otimes E}$ the connection and the Hermitian metric on the trivial line bundle with fiber $\left(L^{p} \otimes E\right)_{x_{0}}$ induced by this trivialization. Let $\Gamma^{L}, \Gamma^{E}$ be the connection forms of $\nabla^{L}$ and $\nabla^{E}$ with respect to some fixed frames for $L$, $E$ which is parallel along the curve $\gamma_{Z}:[0,1] \ni u \rightarrow \exp _{x_{0}}^{X}(u Z)$ under our trivialization of $B^{T_{x_{0}} X}(0, \varepsilon)$. Then we have

$$
\nabla_{U}^{L^{p}}=\nabla_{U}+p \Gamma^{L}(U)+\Gamma^{E}(U)
$$


For any $x_{0} \in X$, fix an orthonormal basis $e_{1}, \ldots, e_{2 n}$ in $T_{x_{0}} X$. We still denote by $\left\{e_{j}\right\}_{j=1}^{2 n}$ the constant vector fields $e_{j}(Z)=e_{j}$ on $B^{T_{x_{0}} X}(0, \varepsilon)$. One can show that the restriction of the norm $\|\cdot\|_{p, m}$ to $\mathscr{C}_{c}^{\infty}\left(B^{T_{x_{0}} X}(0, \varepsilon), L^{p} \otimes E\right) \cong \mathscr{C}_{c}^{\infty}\left(B^{X}\left(x_{0}, \varepsilon\right), L^{p} \otimes E\right)$ is equivalent uniformly on $x_{0} \in X$ and $p \in \mathbb{N}^{*}$ to the norm $\|\cdot\|_{p, m}^{\prime}$ given for $u \in \mathscr{C}_{c}^{\infty}\left(B^{T_{x_{0}} X}(0, \varepsilon), L^{p} \otimes E\right)$ by

$$
\|u\|_{p, m}^{\prime}=\left(\sum_{\ell=0}^{m} \sum_{j_{1}, \ldots, j_{\ell}=1}^{2 n} \int_{T_{x_{0}} X}\left(\frac{1}{\sqrt{p}}\right)^{\ell}\left|\nabla_{e_{j_{1}}}^{L^{p} \otimes E} \cdots \nabla_{e_{j_{\ell}}}^{L^{p} \otimes E} u\right|^{2} d Z\right)^{1 / 2} .
$$

That is, there exists $C_{m}>0$ such that, for any $x_{0} \in X, p \in \mathbb{N}^{*}$ we have

$$
C_{m}^{-1}\|u\|_{p, m}^{\prime} \leq\|u\|_{p, m} \leq C_{m}\|u\|_{p, m}^{\prime}
$$

for any $u \in \mathscr{C}_{c}^{\infty}\left(B^{T_{x_{0}} X}(0, \varepsilon), L^{p} \otimes E\right) \cong \mathscr{C}_{c}^{\infty}\left(B^{X}\left(x_{0}, \varepsilon\right), L^{p} \otimes E\right)$. By choosing an appropriate covering of $X$ by normal coordinate charts, we can reduce our considerations to the local setting. Without loss of generality, we can assume that $u \in \mathscr{C}_{c}^{\infty}\left(B^{T_{x_{0}} X}(0, \varepsilon), L^{p} \otimes E\right)$ for some $x_{0} \in X$ and the Sobolev norm of $u$ is given by the norm $\|u\|_{p, m}^{\prime}$ given by (3.34). (Later on, we omit 'prime' for simplicity.) We have to show the estimate (3.32), uniform on $x_{0}$. That is, we claim that, for any $m \in \mathbb{N}$, there exists $C_{m}>0$ such that for any $p \in \mathbb{N}^{*}, p \geq p_{0}, \lambda \in \delta, y \in X$, $|\alpha|<c \sqrt{p}$ and $x_{0} \in X$

$$
\left\|\left(\lambda-\frac{1}{p} \Delta_{p ; \alpha, y}\right)^{-1} u\right\|_{p, m+1} \leq C_{m}\|u\|_{p, m}, \quad u \in \mathscr{C}_{c}^{\infty}\left(B^{T_{x_{0}} X}(0, \varepsilon), L^{p} \otimes E\right) .
$$

Proposition 3.5. For any $1 \leq j_{1} \leq \ldots \leq j_{k} \leq 2 n$, the iterated commutator

$$
\left[\frac{1}{\sqrt{p}} \nabla_{e_{j_{1}}}^{L^{p} \otimes E},\left[\frac{1}{\sqrt{p}} \nabla_{e_{j_{2}}}^{L^{p} \otimes E}, \ldots,\left[\frac{1}{\sqrt{p}} \nabla_{e_{j_{k}}}^{L^{p} \otimes E}, \frac{1}{p} \Delta_{p ; \alpha, y}\right] \ldots\right]\right]
$$

defines a family of second order differential operators, bounded uniformly on $p \in \mathbb{N}^{*}, y \in X$, $|\alpha|<c \sqrt{p}$ and $x_{0} \in X$. In particular, there exists $C>0$ such that for $p \in \mathbb{N}^{*},|\alpha|<c \sqrt{p}, y \in X$ and $u, u^{\prime} \in \mathscr{C}_{c}^{\infty}\left(B^{T_{x_{0}} X}(0, \varepsilon), L^{p} \otimes E\right)$

$$
\begin{aligned}
&\left|\left\langle\left[\frac{1}{\sqrt{p}} \nabla_{e_{j_{1}}}^{L^{p} \otimes E},\left[\frac{1}{\sqrt{p}} \nabla_{e_{j_{2}}}^{L^{p} \otimes E}, \ldots,\left[\frac{1}{\sqrt{p}} \nabla_{e_{j_{k}}}^{L^{p} \otimes E}, \frac{1}{p} \Delta_{p ; \alpha, y}\right] \ldots\right]\right] u, u^{\prime}\right\rangle_{p, 0}\right| \\
& \leq C\|u\|_{p, 1}\left\|u^{\prime}\right\|_{p, 1}
\end{aligned}
$$

Proof. By (3.15), (3.17) and (3.19), the operator $\frac{1}{p} \Delta_{p ; \alpha, y}$ has the form

$$
\begin{array}{r}
\frac{1}{p} \Delta_{p ; \alpha, y}=\sum_{i, j} a_{p ; \alpha, y}^{i j}(Z)\left(\frac{1}{\sqrt{p}} \nabla_{e_{i}}^{L^{p} \otimes E}\right)\left(\frac{1}{\sqrt{p}} \nabla_{e_{j}}^{L^{p} \otimes E}\right) \\
+\sum_{\ell} b_{p ; \alpha, y}^{\ell}(Z) \frac{1}{\sqrt{p}} \nabla_{e_{\ell}}^{L^{p} \otimes E}+c_{p ; \alpha, y}(Z),
\end{array}
$$

where

$$
\begin{aligned}
& a_{p ; \alpha, y}^{i j}(Z)=-g^{i j}(Z), \\
& b_{p ; \alpha, y}^{\ell}(Z)=\frac{1}{\sqrt{p}} \sum_{j, k=1}^{2 n} g^{j k}(Z) \Gamma_{j k}^{\ell}(Z)-\frac{2 \alpha}{\sqrt{p}} \sum_{j=1}^{2 n} g^{j \ell}(Z) e_{j}\left(\widetilde{d}_{p, y}\right),
\end{aligned}
$$




$$
\begin{aligned}
c_{p ; \alpha, y}(Z)= & -\tau(Z)-\frac{\alpha}{p} \sum_{j, k=1}^{2 n} g^{j k}(Z)\left(e_{j}\left(e_{k}\left(\widetilde{d}_{p, y}\right)\right)-\Gamma_{j k}^{\ell}(Z) e_{\ell}\left(\tilde{d}_{p, y}\right)\right) \\
& -\frac{\alpha^{2}}{p} \sum_{j, k=1}^{2 n} g^{j k}(Z) e_{j}\left(\widetilde{d}_{p, y}\right) e_{k}\left(\widetilde{d}_{p, y}\right) .
\end{aligned}
$$

It is easy to see that if $f_{p ; \alpha, y}$ is $a_{p ; \alpha, y}^{i j}, b_{p ; \alpha, y}^{\ell}$ or $c_{p ; \alpha, y}$, the iterated commutator

$$
\left[\frac{1}{\sqrt{p}} \nabla_{e_{j_{1}}}^{L^{p} \otimes E},\left[\frac{1}{\sqrt{p}} \nabla_{e_{j_{2}}}^{L^{p} \otimes E}, \ldots,\left[\frac{1}{\sqrt{p}} \nabla_{e_{j_{k}}}^{L^{p} \otimes E}, f_{p ; \alpha, y}\right] \ldots\right]\right]
$$

is a smooth function on $B^{T_{x_{0}} X}(0, \varepsilon)$ with sup-norm, uniformly bounded on $p \in \mathbb{N}^{*}, y \in X$, $|\alpha|<c \sqrt{p}$ and $x_{0} \in X$.

Recall the commutator relations

$$
\left[\frac{1}{\sqrt{p}} \nabla_{e_{i}}^{L^{p} \otimes E}, \frac{1}{\sqrt{p}} \nabla_{e_{j}}^{L^{p} \otimes E}\right]=\frac{1}{p} R^{L^{p} \otimes E}\left(e_{i}, e_{j}\right)=R^{L}\left(e_{i}, e_{j}\right)+\frac{1}{p} R^{E}\left(e_{i}, e_{j}\right) .
$$

Using these facts, one can see that the iterated commutator (3.37) has the same structure as $\frac{1}{p} \Delta_{p ; \alpha, y}$. This easily completes the proof.

Now the proof of (3.36) is completed as in [10, Theorem 4.10] or [23, Theorem 1.9]. For any $1 \leq j_{1} \leq \ldots \leq j_{\ell} \leq 2 n$ with $\ell=1, \ldots, m$, we can write the operator

$$
\left(\frac{1}{\sqrt{p}} \nabla_{e_{j_{1}}}^{L^{p} \otimes E}\right)\left(\frac{1}{\sqrt{p}} \nabla_{e_{j_{2}}}^{L^{p} \otimes E}\right) \ldots\left(\frac{1}{\sqrt{p}} \nabla_{e_{j_{\ell}}}^{L^{p} \otimes E}\right)\left(\lambda-\frac{1}{p} \Delta_{p ; \alpha, y}\right)^{-1}
$$

as a linear combination of operators of the type

$$
\begin{aligned}
{\left[\frac{1}{\sqrt{p}} \nabla_{e_{j_{1}}}^{L^{p} \otimes E},\left[\frac{1}{\sqrt{p}} \nabla_{e_{j_{2}}}^{L^{p} \otimes E}, \ldots,\left[\frac{1}{\sqrt{p}} \nabla_{e_{j_{k}}}^{L^{p} \otimes E},\left(\lambda-\frac{1}{p} \Delta_{p ; \alpha, y}\right)^{-1}\right] \ldots\right]\right] \times } & \\
& \times\left(\frac{1}{\sqrt{p}} \nabla_{e_{j_{k+1}}}^{L^{p} \otimes E}\right) \ldots\left(\frac{1}{\sqrt{p}} \nabla_{e_{j_{\ell}}}^{L^{p} \otimes E}\right)
\end{aligned}
$$

and of the operator

$$
\left(\lambda-\frac{1}{p} \Delta_{p ; \alpha, y}\right)^{-1}\left(\frac{1}{\sqrt{p}} \nabla_{e_{j_{1}}}^{L^{p} \otimes E}\right)\left(\frac{1}{\sqrt{p}} \nabla_{e_{j_{2}}}^{L^{p} \otimes E}\right) \ldots\left(\frac{1}{\sqrt{p}} \nabla_{e_{j_{\ell}}}^{L^{p} \otimes E}\right) .
$$

Each commutator

$$
\left[\frac{1}{\sqrt{p}} \nabla_{e_{j_{1}}}^{L^{p} \otimes E},\left[\frac{1}{\sqrt{p}} \nabla_{e_{j_{2}}}^{L^{p} \otimes E}, \ldots,\left[\frac{1}{\sqrt{p}} \nabla_{e_{j_{k}}}^{L^{p} \otimes E},\left(\lambda-\frac{1}{p} \Delta_{p ; \alpha, y}\right)^{-1}\right] \ldots\right]\right]
$$

is a linear combination of operators of the form

$$
\left(\lambda-\frac{1}{p} \Delta_{p ; \alpha, y}\right)^{-1} R_{1}\left(\lambda-\frac{1}{p} \Delta_{p ; \alpha, y}\right)^{-1} R_{2} \ldots R_{k}\left(\lambda-\frac{1}{p} \Delta_{p ; \alpha, y}\right)^{-1},
$$

where the operators $R_{1}, \ldots, R_{k}$ are of the form

$$
\left[\frac{1}{\sqrt{p}} \nabla_{e_{i_{1}}}^{L^{p} \otimes E},\left[\frac{1}{\sqrt{p}} \nabla_{e_{i_{2}}}^{L^{p} \otimes E}, \ldots,\left[\frac{1}{\sqrt{p}} \nabla_{e_{i_{l}}}^{L^{p} \otimes E}, \frac{1}{p} \Delta_{p ; \alpha, y}\right] \ldots\right] .\right.
$$

By Proposition 3.5, each operator $R_{j}$ defines a bounded operator from $H^{1}$ to $H^{-1}$ with the norm, uniformly bounded on $p \in \mathbb{N}^{*},|\alpha|<c \sqrt{p}, y \in X$. Therefore, by Theorem 3.2 , each 
operator (3.44) defines a bounded operator from $L^{2}$ to $H^{1}$ with the norm, uniformly bounded on $p \in \mathbb{N}^{*}, p \geq p_{0},|\alpha|<c \sqrt{p}, y \in X$. This immediately completes the proof.

3.4. Pointwise exponential estimates for the resolvents. In this section, we derive the pointwise estimates for the Schwartz kernel $R_{\lambda, p}^{(m)}(\cdot, \cdot) \in \mathscr{C}^{-\infty}\left(X \times X, \pi_{1}^{*}\left(L^{p} \otimes E\right) \otimes \pi_{2}^{*}\left(L^{p} \otimes E\right)^{*}\right)$ of the operator $\left(\lambda-\frac{1}{p} \Delta_{p}\right)^{-m}$. Recall that $p_{0} \in \mathbb{N}^{*}, c>0$ are given in Theorems 2.6, 3.2,

Theorem 3.6. For any $m, k \in \mathbb{N}$ with $m>2 n+k+1$, for any $p \in \mathbb{N}^{*}, p \geq p_{0}$, and $\lambda \in \delta$, we have $R_{\lambda, p}^{(m)}(\cdot, \cdot) \in \mathscr{C}^{k}\left(X \times X, \pi_{1}^{*}\left(L^{p} \otimes E\right) \otimes \pi_{2}^{*}\left(L^{p} \otimes E\right)^{*}\right)$ and, for any $c_{1} \in(0, c)$, there exists $C_{m, k}>0$ such that for any $p \in \mathbb{N}^{*}, p \geq p_{0}, \lambda \in \delta, x, x^{\prime} \in X$, we have

$$
\left|R_{\lambda, p}^{(m)}\left(x, x^{\prime}\right)\right|_{C^{k}} \leq C_{m, k} p^{n+\frac{k}{2}} e^{-c_{1} \sqrt{p} d\left(x, x^{\prime}\right)} .
$$

Proof. By (3.29), for any $m \in \mathbb{N}, p \in \mathbb{N}^{*}, p \geq p_{0}, \lambda \in \delta, y \in X$ and $|\alpha|<c \sqrt{p}$, we have

$$
f_{\alpha, p, y}\left(\lambda-\frac{1}{p} \Delta_{p}\right)^{-m} f_{\alpha, p, y}^{-1}=\left(\lambda-\frac{1}{p} \Delta_{p ; \alpha, y}\right)^{-m} .
$$

As in Remark 3.3, one can show that this formula gives a well-defined operator from $\mathscr{C}_{c}^{\infty}\left(X, L^{p} \otimes\right.$ $E)$ to $\mathscr{C}^{-\infty}\left(X, L^{p} \otimes E\right)$.

Since $\Delta_{p}$ is formally self-adjoint with respect to $\|\cdot\|_{p, 0}$, we have $\Delta_{p ; \alpha, y}^{*}=\Delta_{p ;-\alpha, y}$. Using this fact and Theorem 3.4, we easily get that, for any $m_{1} \in \mathbb{Z}$, there exists $C_{m, m_{1}}>0$ such that, for all $p \in \mathbb{N}^{*}, p \geq p_{0}, \lambda \in \delta, y \in X$ and $|\alpha|<c \sqrt{p}$, we have

$$
\left\|\left(\lambda-\frac{1}{p} \Delta_{p ; \alpha, y}\right)^{-m}\right\|_{p}^{m_{1}, m_{1}+m} \leq C_{m, m_{1}} .
$$

The Schwartz kernel $R_{\lambda, p ; \alpha, y}^{(m)}(\cdot, \cdot) \in \mathscr{C}^{k}\left(X \times X, \pi_{1}^{*}\left(L^{p} \otimes E\right) \otimes \pi_{2}^{*}\left(L^{p} \otimes E\right)^{*}\right)$ of the operator $\left(\lambda-\frac{1}{p} \Delta_{p ; \alpha, y}\right)^{-m}$ is related to the Schwartz kernel $R_{\lambda, p}^{(m)}(\cdot, \cdot)$ of the operator $\left(\lambda-\frac{1}{p} \Delta_{p}\right)^{-m}$ by the formula

$$
R_{\lambda, p ; \alpha, y}^{(m)}\left(x, x^{\prime}\right)=e^{\alpha \widetilde{d}_{p, y}(x)} R_{\lambda, p}^{(m)}\left(x, x^{\prime}\right) e^{-\alpha \widetilde{d}_{p, y}\left(x^{\prime}\right)}, \quad x, x^{\prime} \in X .
$$

For $x, x^{\prime}, y \in X$ and $v \in\left(L^{p} \otimes E\right)_{x^{\prime}}$, we can write

$$
e^{\alpha \widetilde{d}_{p, y}(x)} R_{\lambda, p}^{(m)}\left(x, x^{\prime}\right) e^{-\alpha \widetilde{d}_{p, y}\left(x^{\prime}\right)} v=\left(\left(\lambda-\frac{1}{p} \Delta_{p ; \alpha, y}\right)^{-m} \delta_{v}\right)(x) \in\left(L^{p} \otimes E\right)_{x} .
$$

In particular, putting $y=x^{\prime}$, we get for $x, x^{\prime} \in X$ and $v \in\left(L^{p} \otimes E\right)_{x^{\prime}}$,

$$
e^{\alpha \widetilde{d}_{p, x^{\prime}}(x)} R_{\lambda, p}^{(m)}\left(x, x^{\prime}\right) e^{-\alpha \widetilde{d}_{p, x^{\prime}}\left(x^{\prime}\right)} v=\left(\left(\lambda-\frac{1}{p} \Delta_{p ; \alpha, x^{\prime}}\right)^{-m} \delta_{v}\right)(x) \in\left(L^{p} \otimes E\right)_{x} .
$$

By (3.9), it follows that, for $0<\alpha<c \sqrt{p}$ and $x^{\prime} \in X$, we have an estimate $e^{\alpha \widetilde{d}_{p, x^{\prime}}\left(x^{\prime}\right)} \leq e^{c}$. It is in this place that we need to use the smoothed distance function $\widetilde{d}$, depending on $p$. Assuming $0<\alpha<c \sqrt{p}$, by Propositions 2.2, 2.3, and (3.51), we get, for $m>2 n+1$, that 
$R_{\lambda, p}^{(m)}\left(x, x^{\prime}\right)$ is continuous and

$$
\begin{aligned}
\sup _{x, x^{\prime} \in X} e^{\alpha \widetilde{d}_{p, x^{\prime}}(x)}\left|R_{\lambda, p}^{(m)}\left(x, x^{\prime}\right)\right| & \leq e^{c} \sup _{v \in\left(L^{p} \otimes E\right)_{x^{\prime}},|v|=1}\left\|\left(\lambda-\frac{1}{p} \Delta_{p ; \alpha, x^{\prime}}\right)^{-m} \delta_{v}\right\|_{\mathscr{b}_{b}^{0}} \\
& \leq C_{1} p^{n / 2} \sup _{v \in\left(L^{p} \otimes E\right)_{x^{\prime}},|v|=1}\left\|\left(\lambda-\frac{1}{p} \Delta_{p ; \alpha, x^{\prime}}\right)^{-m} \delta_{v}\right\|_{p, n+1} \\
& \leq C_{2} p^{n / 2} \sup _{v \in\left(L^{p} \otimes E\right)_{x^{\prime}},|v|=1}\left\|\delta_{v}\right\|_{p, n+1-m} \leq C_{3} p^{n} .
\end{aligned}
$$

Similarly, for any $Q_{1} \in B D^{k_{1}}\left(X, L^{p} \otimes E\right)$ and $Q_{2} \in B D^{k_{2}}\left(X, L^{p} \otimes E\right), k_{1}+k_{2}=k$, by the argument after (3.14), we get with $m>2 n+k+1$,

$$
\begin{aligned}
\sup _{x, x^{\prime} \in X} e^{\alpha \widetilde{d}_{p, x^{\prime}}(x)} \mid & \left(Q_{1} \otimes Q_{2}\right) R_{\lambda, p}^{(m)}\left(x, x^{\prime}\right) \mid \\
& \leq e^{c} \sup _{v \in\left(L^{p} \otimes E\right)_{x^{\prime}},|v|=1}\left\|F_{\alpha, p, x^{\prime}} Q_{1}\left(\lambda-\frac{1}{p} \Delta_{p}\right)^{-m} Q_{2}^{*} F_{\alpha, p, x^{\prime}}^{-1} \delta_{v}\right\|_{\mathscr{C}_{b}^{0}} \\
& =e^{c} \sup _{v \in\left(L^{p} \otimes E\right)_{x^{\prime}},|v|=1}\left\|Q_{1 ; \alpha, p, x^{\prime}}\left(\lambda-\frac{1}{p} \Delta_{p ; \alpha, x^{\prime}}\right)^{-m} Q_{2 ; \alpha, p, x^{\prime}}^{*} \delta_{v}\right\|_{\mathscr{C}_{b}^{0}} \\
& \leq C_{1} p^{n / 2} \sup _{v \in\left(L^{p} \otimes E\right)_{x^{\prime}},|v|=1}\left\|Q_{1 ; \alpha, p, x^{\prime}}\left(\lambda-\frac{1}{p} \Delta_{p ; \alpha, x^{\prime}}\right)^{-m} Q_{2 ; \alpha, p, x^{\prime}}^{*} \delta_{v}\right\|_{p, n+1} \\
\leq & C_{2} p^{\left(n+k_{1}\right) / 2} \sup _{v \in\left(L^{p} \otimes E\right)_{x^{\prime}},|v|=1}\left\|\left(\lambda-\frac{1}{p} \Delta_{p ; \alpha, x^{\prime}}\right)^{-m} Q_{2 ; \alpha, p, x^{\prime}}^{*} \delta_{v}\right\|_{p, n+k_{1}+1} \\
\leq & C_{3} p^{\left(n+k_{1}\right) / 2} \sup _{v \in\left(L^{p} \otimes E\right)_{x^{\prime}},|v|=1}\left\|Q_{2 ; \alpha, p, x^{\prime}}^{*} \delta_{v}\right\|_{p, n-m+k_{1}+1} \\
\leq & C_{4} p^{(n+k) / 2} \sup _{v \in\left(L^{p} \otimes E\right)_{x^{\prime}},|v|=1}\left\|\delta_{v}\right\|_{p, n-m+k+1} \leq C_{5} p^{n+k / 2} .
\end{aligned}
$$

For any $x_{0} \in X$, fix an orthonormal basis $e_{1}, \ldots, e_{2 n}$ in $T_{x_{0}} X$. As above, we extend it to a frame $e_{1}, \ldots, e_{2 n}$ on $B^{X}(0, \varepsilon)$ as constant vector fields on $T_{x_{0}} X$. One can show (see, for instance, [14, Proposition 1.5]) that the norm $\|\cdot\|_{\mathscr{C}_{b}^{k}}$ on $\mathscr{C}_{b}^{k}\left(X, L^{p} \otimes E\right)$ is equivalent uniformly on $p \in \mathbb{N}^{*}$ to the norm $\|\cdot\|_{\mathscr{C}_{b}^{k}}^{\prime}$ given for $u \in \mathscr{C}_{b}^{k}\left(X, L^{p} \otimes E\right)$ by

$$
\|u\|_{\mathscr{C}_{b}^{k}}^{\prime}=\sup _{x_{0} \in X} \sup _{0 \leq \ell \leq k}\left\|\nabla_{e_{j_{1}}}^{L^{p} \otimes E} \cdots \nabla_{e_{j_{\ell}}}^{L^{p} \otimes E} u\left(x_{0}\right)\right\| .
$$

That is, there exists $C_{k}>0$ such that, for any $p \in \mathbb{N}^{*}$ and $u \in \mathscr{C}_{b}^{k}\left(X, L^{p} \otimes E\right)$, we have

$$
C_{k}^{-1}\|u\|_{\mathscr{C}_{b}^{k}} \leq\|u\|_{\mathscr{C}_{b}^{k}}^{\prime} \leq C_{k}\|u\|_{\mathscr{C}_{b}^{k}}
$$

Let $\phi \in \mathscr{C}_{c}^{\infty}\left(\mathbb{R}^{2 n}\right)$ be any function supported in the ball $B(0, \varepsilon)$ such that $\phi \equiv 1$ on $B(0, \varepsilon / 2)$. Consider the function $\phi_{x_{0}} \in \mathscr{C}_{c}^{\infty}\left(B^{X}\left(x_{0}, \varepsilon\right)\right)$, corresponding to $\phi$ under the isomorphisms $\mathscr{C}_{c}^{\infty}(B(0, \varepsilon)) \cong \mathscr{C}_{c}^{\infty}\left(B^{T_{x_{0}} X}(0, \varepsilon)\right) \cong \mathscr{C}_{c}^{\infty}\left(B^{X}\left(x_{0}, \varepsilon\right)\right)$ induced by the basis $e_{1}, \ldots, e_{2 n}$ and the exponential map $\exp _{x_{0}}^{X}$. The family $\left\{\phi_{x_{0}}, x_{0} \in X\right\}$ is bounded in $\mathscr{C}_{b}^{\infty}(X)$.

By (3.55), it follows that there exists $C_{k}>0$ such that, for any $x, x^{\prime} \in X$, we have

$$
\left|R_{\lambda, p}^{(m)}\left(x, x^{\prime}\right)\right|_{\mathscr{C}^{k}} \leq C_{k} \sup _{Q_{1}, Q_{2}}\left|\left(Q_{1} \otimes Q_{2}\right) R_{\lambda, p}^{(m)}\left(x, x^{\prime}\right)\right|
$$


with the supremum taken over all pairs $\left(Q_{1}, Q_{2}\right)$, where $Q_{1} \in B D^{k_{1}}\left(X, L^{p} \otimes E\right)$ and $Q_{2} \in$ $B D^{k_{2}}\left(X, L^{p} \otimes E\right), k_{1}+k_{2} \leq k$, have the form

$$
Q_{1}=\nabla_{e_{i_{1}}}^{L^{p} \otimes E} \cdots \nabla_{e_{i_{1}}}^{L^{p} \otimes E} \phi_{x}, \quad Q_{2}=\nabla_{e_{j_{1}}}^{L^{p} \otimes E} \cdots \nabla_{e_{j_{k_{2}}}}^{L^{p} \otimes E} \phi_{x^{\prime}}
$$

and $i_{1}, \ldots, i_{k_{1}}, j_{1}, \ldots, j_{k_{2}} \in\{1, \ldots, 2 n\}$, that immediately completes the proof of (3.46).

3.5. Proof of Theorems 1.2 and 1.3. In this section, we complete the proofs of Theorems 1.2 and 1.3 .

Proof of Theorem 1.2 Let $k \in \mathbb{N}$. Take an arbitrary $m>2 n+k+1$. By (3.1), we have

$$
P_{p}\left(x, x^{\prime}\right)=\frac{1}{2 \pi i} \int_{\delta} \lambda^{m-1} R_{\lambda, p}^{(m)}\left(x, x^{\prime}\right) d \lambda .
$$

By Theorem 3.6, it implies immediately the $\mathscr{C}^{k}$-estimate in Theorem 1.2 with $c>0$ given by that theorem.

Proof of Theorem 1.3 First, we observe that, for $\widetilde{\tau} \in \mathscr{C}^{\infty}(\tilde{X})$ and $\tau \in \mathscr{C}^{\infty}(X)$ given by (1.6), we have $\widetilde{\tau}=\pi^{*} \tau$. Next, the quantities $\widetilde{\mu}_{0}$ and $\mu_{0}$ defined by (1.7) are equal. In particular, $\widetilde{\mu}_{0}>0$. By Theorem 1.1, there exists constant $C_{L}>0$ such that for any $p \in \mathbb{N}^{*}$

$$
\sigma\left(\Delta_{p}\right) \cup \sigma\left(\widetilde{\Delta}_{p}\right) \subset\left[-C_{L}, C_{L}\right] \cup\left[2 p \mu_{0}-C_{L},+\infty\right) .
$$

Recall that $\delta$ denotes the counterclockwise oriented circle in $\mathbb{C}$ centered at 0 of radius $\mu_{0}$.

For any $p, m \in \mathbb{N}, p \geq p_{0}$, and $\lambda \in \delta$, denote by $\widetilde{R}_{\lambda, p}^{(m)} \in \mathscr{C}^{-\infty}\left(\widetilde{X} \times \widetilde{X}, \pi_{1}^{*}\left(\widetilde{L}^{p} \otimes \widetilde{E}\right) \otimes \pi_{2}^{*}\left(\widetilde{L}^{p} \otimes \widetilde{E}\right)^{*}\right)$ and $R_{\lambda, p}^{(m)} \in \mathscr{C}^{-\infty}\left(X \times X, \pi_{1}^{*}\left(L^{p} \otimes E\right) \otimes \pi_{2}^{*}\left(L^{p} \otimes E\right)^{*}\right)$ the Schwartz kernels of the operators $\left(\lambda-\frac{1}{p} \widetilde{\Delta}_{p}\right)^{-m}$ and $\left(\lambda-\frac{1}{p} \Delta_{p}\right)^{-m}$, respectively. Recall that, for any $x^{\prime}$, they satisfy the identities

$$
\left(\lambda-\frac{1}{p} \widetilde{\Delta}_{p}\right)^{m} \widetilde{R}_{\lambda, p}^{(m)}\left(\cdot, x^{\prime}\right)=\delta_{x^{\prime}}, \quad\left(\lambda-\frac{1}{p} \Delta_{p}\right)^{m} R_{\lambda, p}^{(m)}\left(\cdot, x^{\prime}\right)=\delta_{x^{\prime}} .
$$

Moreover, $u=R_{\lambda, p}^{(m)}\left(\cdot, x^{\prime}\right)$ is the unique distributional solution of the equation

$$
\left(\lambda-\frac{1}{p} \Delta_{p}\right)^{m} u=\delta_{x^{\prime}}
$$

Let $m>2 n+1$. Then, by elliptic regularity and Sobolev embedding theorem, $\widetilde{R}_{\lambda, p}^{(m)}$ and $R_{\lambda, p}^{(m)}$ are continuous. We claim that there exists $p_{1} \in \mathbb{N}$ such that for any $p>p_{1}$ and $x, x^{\prime} \in \tilde{X}$,

$$
\sum_{\gamma \in \Gamma} \widetilde{R}_{\lambda, p}^{(m)}\left(\gamma x, x^{\prime}\right)=R_{\lambda, p}^{(m)}\left(\pi(x), \pi\left(x^{\prime}\right)\right)
$$

By [28], there exists $K>0$ such that $\sum_{\gamma \in \Gamma} e^{-a d\left(\gamma x, x^{\prime}\right)}<+\infty$ for any $a>K$ and $x, x^{\prime} \in \tilde{X}$. Put $p_{1} \geq K^{2} / c^{2}+p_{0}$. Then, by Theorem 3.6, for any $p>p_{1}$ and $x, x^{\prime} \in \widetilde{X}$, the series in the left-hand side of (3.62) is absolutely convergent with respect to $\mathscr{C}^{0}$-norm and its sum

$$
\widetilde{S}_{\lambda, p}^{(m)}\left(x, x^{\prime}\right)=\sum_{\gamma \in \Gamma} \widetilde{R}_{\lambda, p}^{(m)}\left(\gamma x, x^{\prime}\right)
$$

is a $\Gamma$-invariant continuous section on $\widetilde{X} \times \widetilde{X}$. So we can write

$$
\widetilde{S}_{\lambda, p}^{(m)}\left(x, x^{\prime}\right)=S_{\lambda, p}^{(m)}\left(\pi(x), \pi\left(x^{\prime}\right)\right) \text { with } S_{\lambda, p}^{(m)} \in \mathscr{C}^{0}\left(X \times X, \pi_{1}^{*}\left(L^{p} \otimes E\right) \otimes \pi_{2}^{*}\left(L^{p} \otimes E\right)^{*}\right) .
$$


Moreover, by (3.60), for any $x^{\prime} \in \widetilde{X}, \widetilde{S}_{\lambda, p}^{(m)}\left(\cdot, x^{\prime}\right)$ satisfies the identity

$$
\left(\lambda-\frac{1}{p} \widetilde{\Delta}_{p}\right)^{m} \widetilde{S}_{\lambda, p}^{(m)}\left(\cdot, x^{\prime}\right)=\delta_{x^{\prime}}
$$

Therefore, for any $x^{\prime} \in X, S_{\lambda, p}^{(m)}\left(\cdot, x^{\prime}\right)$ satisfies the identity

$$
\left(\lambda-\frac{1}{p} \Delta_{p}\right)^{m} S_{\lambda, p}^{(m)}\left(\cdot, x^{\prime}\right)=\delta_{x^{\prime}}
$$

By the uniqueness of the solution of (3.61), it follows that $S_{\lambda, p}^{(m)}=R_{\lambda, p}^{(m)}$. This completes the proof of (3.62).

Since, by Theorem 3.6, the series in the left-hand side of (3.62) is absolutely convergent with respect to the $\mathscr{C}^{0}$-norm uniformly in $\lambda \in \delta$, we can integrate it term by term over $\delta$. Using (3.58) and (3.62), for any $p>p_{1}$ and $x, x^{\prime} \in \widetilde{X}$, we get

$$
\begin{aligned}
\sum_{\gamma \in \Gamma} \widetilde{P}_{p}\left(\gamma x, x^{\prime}\right) & =\frac{1}{2 \pi i} \sum_{\gamma \in \Gamma} \int_{\delta} \lambda^{m-1} \widetilde{R}_{\lambda, p}^{(m)}\left(\gamma x, x^{\prime}\right) d \lambda \\
& =\frac{1}{2 \pi i} \int_{\delta} \lambda^{m-1} R_{\lambda, p}^{(m)}\left(\pi(x), \pi\left(x^{\prime}\right)\right) d \lambda=P_{p}\left(\pi(x), \pi\left(x^{\prime}\right)\right) .
\end{aligned}
$$

The proof of Theorem 1.3 is completed.

\section{FULL OFF-DIAGONAL ASYMPTOTIC EXPANSION}

In this section, we study the full off-diagonal asymptotic expansion in the geometric situation of our article described in the Introduction.

In the case of a compact Kähler manifold the asymptotic expansion of the Bergman kernel $P_{p}(x, x)$ restricted to the diagonal was initiated by Tian [29], who proved the expansion up to first order. Catlin [8] and Zelditch [30] proved the asymptotic expansion of $P_{p}(x, x)$ up to arbitrary order, see [22] for the numerous applications of these results.

On the other hand, the off-diagonal expansion of the Bergman kernel has many applications. In the case of complex manifolds the expansion of $P_{p}\left(x, x^{\prime}\right)$ holds in a fixed neighborhood of the diagonal (independent of $p$ ), see [10], [22, Theorem 4.2.1]. Such kind of expansion is called full off-diagonal expansion. As already noted in [22, Problem 6.1, p. 292] the proof of the full off-diagonal expansion holds also for complex manifolds with bounded geometry.

In the case of the Bergman kernel associated to the renormalized Bochner-Laplacian considered in the present article, it was shown in [23, Theorem 1.19] that the off-diagonal expansion holds in a neighborhood of size $1 / \sqrt{p}$ of the diagonal. This is called near off-diagonal expansion. Moreover, it was shown in [22, p. 329] that the Bergman kernel is $\mathcal{O}\left(p^{-\infty}\right)$ outside a neighborhood of size $p^{-\theta}$, for any $\theta \in(0,1 / 2)$. These estimates are used in the proof of the Kodaira embedding theorem for symplectic manifolds [23, Theorem 3.6]. In [17] a less precise estimate than [23, Theorem 1.19] was obtained in a neighborhood of size $p^{-\theta}$, $\theta \in(0,1 / 2)$, which is however enough to derive the Berezin-Toeplitz quantization for the quantum spaced $\mathcal{H}_{p}$ in [12]. Finally, in [15] the full off-diagonal expansion for the Bergman kernel associated to the renormalized Bochner-Laplacian was proved by combining [23, $\S 1]$ and weight function trick in [14]. 
In this section we extend the results on the full off-diagonal asymptotic expansion to the case of manifolds of bounded geometry. Moreover, using the technique of weighted estimates of the previous sections, we slightly improve the remainder in the asymptotic expansions. We will keep the setting described in Introduction.

Consider the fiberwise product $T X \times_{X} T X=\left\{\left(Z, Z^{\prime}\right) \in T_{x_{0}} X \times T_{x_{0}} X: x_{0} \in X\right\}$. Let $\pi$ : $T X \times_{X} T X \rightarrow X$ be the natural projection given by $\pi\left(Z, Z^{\prime}\right)=x_{0}$. The kernel $P_{q, p}\left(x, x^{\prime}\right)$ (of the operator $\left.\left(\Delta_{p}\right)^{q} P_{\mathcal{H}_{p}}\right)$ induces a smooth section $P_{q, p, x_{0}}\left(Z, Z^{\prime}\right)$ of the vector bundle $\pi^{*}(\operatorname{End}(E))$ on $T X \times_{X} T X$ defined for all $x_{0} \in X$ and $Z, Z^{\prime} \in T_{x_{0}} X$ with $|Z|,\left|Z^{\prime}\right|<a^{X}$.

We will follow the arguments of [15] and [23]. We will use the normal coordinates near an arbitrary point $x_{0} \in X$ introduced in the proof of Theorem 3.4 . Let $\left\{e_{j}\right\}$ be an oriented orthonormal basis of $T_{x_{0}} X$. It gives rise to an isomorphism $X_{0}:=\mathbb{R}^{2 n} \cong T_{x_{0}} X$.

Consider the trivial bundles $L_{0}$ and $E_{0}$ with fibers $L_{x_{0}}$ and $E_{x_{0}}$ on $X_{0}$. Recall that we have the Riemannian metric $g^{T X}$ on $B^{T_{x_{0}} X}\left(0, a^{X}\right)$ as well as the connections $\nabla^{L}, \nabla^{E}$ and the Hermitian metrics $h^{L}, h^{E}$ on the restrictions of $L_{0}, E_{0}$ to $B^{T_{x_{0}} X}\left(0, a^{X}\right)$ induced by the identification $B^{T_{x_{0}} X}\left(0, a^{X}\right) \cong B^{X}\left(x_{0}, a^{X}\right)$. In particular, $h^{L}, h^{E}$ are the constant metrics $h^{L_{0}}=$ $h^{L_{x_{0}}}, h^{E_{0}}=h^{E_{x_{0}}}$. For $\varepsilon \in\left(0, a^{X} / 4\right)$, one can extend these geometric objects from $B^{T_{x_{0}} X}(0, \varepsilon)$ to $X_{0} \cong T_{x_{0}} X$ in the following way.

Let $\rho: \mathbb{R} \rightarrow[0,1]$ be a smooth even function such that $\rho(v)=1$ if $|v|<2$ and $\rho(v)=0$ if $|v|>4$. Let $\varphi_{\varepsilon}: \mathbb{R}^{2 n} \rightarrow \mathbb{R}^{2 n}$ be the map defined by $\varphi_{\varepsilon}(Z)=\rho(|Z| / \varepsilon) Z$. We equip $X_{0}$ with the metric $g^{T X_{0}}(Z)=g\left(\varphi_{\varepsilon}(Z)\right)$. Set $\nabla^{E_{0}}=\varphi_{\varepsilon}^{*} \nabla^{E}$. Define the Hermitian connection $\nabla^{L_{0}}$ on $\left(L_{0}, h^{L_{0}}\right)$ by (cf. [10, (4.23)], [23, (1.21)])

$$
\left.\nabla^{L_{0}}\right|_{Z}=\varphi_{\varepsilon}^{*} \nabla^{L}+\frac{1}{2}\left(1-\rho^{2}(|Z| / \varepsilon)\right) R_{x_{0}}^{L}(\mathcal{R}, \cdot),
$$

where $\mathcal{R}(Z)=\sum_{j} Z_{j} e_{j} \in \mathbb{R}^{2 n} \cong T_{Z} X_{0}$.

By [10, (4.24)], [23, (1.22)], if $\varepsilon$ is small enough, then the curvature $R^{L_{0}}$ is positive and satisfies the following estimate for any $x_{0} \in X$,

$$
\inf _{u \in T_{x_{0}} X \backslash\{0\}} \frac{i R^{L_{0}}\left(u, J^{L_{0}} u\right)}{|u|_{g^{T} X}^{2}} \geq \frac{4}{5} \mu_{0} .
$$

Here $J^{L_{0}}$ is the almost complex structure on $X_{0}$ defined by $g^{T X_{0}}$ and $i R^{L_{0}}$. From now on, we fix such an $\varepsilon>0$.

Let $d v_{T X}$ be the Riemannian volume form of $\left(T_{x_{0}} X, g^{T_{x_{0}} X}\right)$. Let $\kappa$ be the smooth positive function on $X_{0}$ defined by the equation

$$
d v_{X_{0}}(Z)=\kappa(Z) d v_{T X}(Z), \quad Z \in X_{0} .
$$

Let $\Delta_{p}^{X_{0}}=\Delta^{L_{0}^{p} \otimes E_{0}}-p \tau_{0}$ be the associated renormalized Bochner-Laplacian acting on $\mathscr{C}^{\infty}\left(X_{0}, L_{0}^{p} \otimes E_{0}\right)$. Then (cf. [23, (1.23)]) there exists $C_{L_{0}}>0$ such that for any $p$

$$
\sigma\left(\Delta_{p}^{X_{0}}\right) \subset\left[-C_{L_{0}}, C_{L_{0}}\right] \cup\left[\frac{8}{5} p \mu_{0}-C_{L_{0}},+\infty\right)
$$

Consider the subspace $\mathcal{H}_{p}^{0}$ in $\mathscr{C}^{\infty}\left(X_{0}, L_{0}^{p} \otimes E_{0}\right) \cong \mathscr{C}^{\infty}\left(\mathbb{R}^{2 n}, E_{x_{0}}\right)$ spanned by the eigensections of $\Delta_{p}^{X_{0}}$ corresponding to eigenvalues in $\left[-C_{L_{0}}, C_{L_{0}}\right]$. Let $P_{\mathcal{H}_{p}^{0}}$ be the orthogonal projection onto $\mathcal{H}_{p}^{0}$. The smooth kernel of $\left(\Delta_{p}^{X_{0}}\right)^{q} P_{\mathcal{H}_{p}^{0}}$ with respect to the Riemannian volume form $d v_{X_{0}}$ 
is denoted by $P_{q, p}^{0}\left(Z, Z^{\prime}\right)$. As proved in [23, Proposition 1.3], the kernels $P_{q, p, x_{0}}\left(Z, Z^{\prime}\right)$ and $P_{q, p}^{0}\left(Z, Z^{\prime}\right)$ are asymptotically close on $B^{T_{x_{0}} X}(0, \varepsilon)$ in the $\mathscr{C}^{\infty}$-topology, as $p \rightarrow \infty$.

In the next theorem, we improve the $\mathcal{O}\left(p^{-\infty}\right)$-estimate for the norms of the difference between $P_{q, p, x_{0}}\left(Z, Z^{\prime}\right)$ and $P_{q, p}^{0}\left(Z, Z^{\prime}\right)$ of [23, Proposition 1.3], proving an exponential decay estimate. This is essentially the main new result of this section.

Theorem 4.1. There exists $c_{0}>0$ such that, for any $k \in \mathbb{N}$, there exists $C_{k}>0$ such that for $p \in \mathbb{N}^{*}, x_{0} \in X$ and $Z, Z^{\prime} \in B^{X_{0}}(0, \varepsilon)$,

$$
\left|P_{q, p, x_{0}}\left(Z, Z^{\prime}\right)-P_{q, p}^{0}\left(Z, Z^{\prime}\right)\right|_{\mathscr{C} k} \leq C_{k} e^{-c_{0} \sqrt{p}}
$$

Proof. As in Section 3.4, $R_{\lambda, p}^{(m)} \in \mathscr{C}^{-\infty}\left(X \times X, \pi_{1}^{*}\left(L^{p} \otimes E\right) \otimes \pi_{2}^{*}\left(L^{p} \otimes E\right)^{*}\right)$ denotes the Schwartz kernel of the operator $\left(\lambda-\frac{1}{p} \Delta_{p}\right)^{-m}$. We also denote by

$$
R_{\lambda, p}^{X_{0},(m)} \in \mathscr{C}^{-\infty}\left(X_{0} \times X_{0}, \pi_{1}^{*}\left(L_{0}^{p} \otimes E_{0}\right) \otimes \pi_{2}^{*}\left(L_{0}^{p} \otimes E_{0}\right)^{*}\right)
$$

the Schwartz kernel of the operator $\left(\lambda-\frac{1}{p} \Delta_{p}^{X_{0}}\right)^{-m}$. For $m>2 n+1$, the distributional sections $R_{\lambda, p}^{(m)}\left(x, x^{\prime}\right)$ and $R_{\lambda, p}^{X_{0},(m)}\left(Z, Z^{\prime}\right)$ are continuous sections.

Recall that $d$ denotes the geodesic distance on $X$ and $d^{X_{0}}$ the geodesic distance on $X_{0}$. By construction, $d$ coincides with $d^{X_{0}}$ on $B^{X}\left(x_{0}, 2 \varepsilon\right) \times B^{X}\left(x_{0}, 2 \varepsilon\right) \cong B^{X_{0}}(0,2 \varepsilon) \times B^{X_{0}}(0,2 \varepsilon)$. Let $\psi \in \mathscr{C}_{c}^{\infty}\left(B^{X_{0}}(0,4 \varepsilon)\right), \psi \equiv 1$ on $B^{X_{0}}(0,3 \varepsilon)$.

Consider a function $\chi \in \mathscr{C}^{\infty}(\mathbb{R})$ such that $\chi(r)=1$ for $|r| \leq \varepsilon$ and $\chi(r)=0$ for $|r| \geq 2 \varepsilon$. For any $\alpha \in \mathbb{R}$ and $W \in X_{0}$, we introduce a weight function $\phi_{\alpha, W}^{X_{0}} \in \mathscr{C}^{\infty}\left(X_{0}\right)$ by

$$
\phi_{\alpha, W}^{X_{0}}(Z)=\exp \left[\alpha \chi\left(d^{X_{0}}(Z, W)\right)\right], \quad Z \in X_{0} .
$$

Consider the operators

$$
\Delta_{p ; \alpha, W}^{X_{0}}:=\phi_{\alpha, W}^{X_{0}} \Delta_{p}^{X_{0}}\left(\phi_{\alpha, W}^{X_{0}}\right)^{-1}
$$

With the same arguments as in Theorem 3.4, one can show that

Theorem 4.2. There exist $c_{0}>0$ and $p_{0} \in \mathbb{N}^{*}$ such that, for any $p \in \mathbb{N}^{*}, p \geq p_{0}, \lambda \in \delta, W \in X_{0}$ and $|\alpha|<c_{0} \sqrt{p}$, the operator $\lambda-\frac{1}{p} \Delta_{p ; \alpha, W}^{X_{0}}$ is invertible in $L^{2}$. Moreover, for any $m \in \mathbb{N}$, the resolvent $\left(\lambda-\frac{1}{p} \Delta_{p ; \alpha, W}^{X_{0}}\right)^{-1}$ maps $H^{m}$ to $H^{m+1}$ with the following norm estimates:

$$
\left\|\left(\lambda-\frac{1}{p} \Delta_{p ; \alpha, W}^{X_{0}}\right)^{-1}\right\|_{p}^{m, m+1} \leq C
$$

where $C>0$ is independent of $p \in \mathbb{N}^{*}, p \geq p_{0}, \lambda \in \delta, W \in X_{0}$ and $x_{0} \in X$. 
For any $Z, W \in B^{X_{0}}(0, \varepsilon / 2)$, we have $d^{X_{0}}(Z, W)<\varepsilon$ and, therefore, $\phi_{\alpha, W}^{X_{0}}(Z)=e^{\alpha}$. For $m>2 n+1$, we have

(4.10)

$$
\begin{aligned}
& \sup _{Z, W \in B^{X_{0}(0, \varepsilon / 2)}}\left|R_{\lambda, p}^{(m)}(Z, W)-R_{\lambda, p}^{X_{0},(m)}(Z, W)\right| \\
& \leq e^{-\frac{1}{2} c_{0} \sqrt{p}} \sup _{Z, W \in B^{X_{0}(0, \varepsilon / 2)}}\left|\phi_{\frac{1}{2} c_{0} \sqrt{p}, W}^{X_{0}}(Z)\left(\psi(Z) R_{\lambda, p}^{(m)}(Z, W)-R_{\lambda, p}^{X_{0},(m)}(Z, W) \psi(W)\right)\right| \\
& \leq e^{-\frac{1}{2} c_{0} \sqrt{p}} \sup _{\substack{W \in B^{X_{0}(0, \varepsilon / 2),} \\
v \in\left(L_{0}^{p} \otimes E_{0}\right)_{W},|v|=1}}\left\|\phi_{\frac{1}{2} c_{0} \sqrt{p}, W}^{X_{0}}\left(\psi\left(\lambda-\frac{1}{p} \Delta_{p}\right)^{-m}-\left(\lambda-\frac{1}{p} \Delta_{p}^{X_{0}}\right)^{-m} \psi\right) \delta_{v}\right\|_{\mathscr{C}_{b}^{0}} \\
& \leq C p^{n / 2} e^{-\frac{1}{2} c_{0} \sqrt{p}} \sup _{\substack{W \in B^{X_{0}(0, \varepsilon / 2),} \\
v \in\left(L_{0}^{p} \otimes E_{0}\right)_{W},|v|=1}}\left\|\phi_{\frac{1}{2} c_{0} \sqrt{p}, W}^{X_{0}}\left(\psi\left(\lambda-\frac{1}{p} \Delta_{p}\right)^{-m}-\left(\lambda-\frac{1}{p} \Delta_{p}^{X_{0}}\right)^{-m} \psi\right) \delta_{v}\right\|_{p, n+1} .
\end{aligned}
$$

By construction, we have for any $u \in \mathscr{C}_{c}^{\infty}\left(B^{X_{0}}(0,2 \varepsilon), E_{x_{0}}\right)$,

$$
\Delta_{p} u(Z)=\Delta_{p}^{X_{0}} u(Z)
$$

Then, for any $u$, we have

$$
\left(\lambda-\frac{1}{p} \Delta_{p}^{X_{0}}\right)^{m} \psi\left(\lambda-\frac{1}{p} \Delta_{p}\right)^{-m} u=\left[\left(\lambda-\frac{1}{p} \Delta_{p}^{X_{0}}\right)^{m}, \psi\right]\left(\lambda-\frac{1}{p} \Delta_{p}\right)^{-m} u+\psi u
$$

and

$$
\begin{aligned}
& \psi\left(\lambda-\frac{1}{p} \Delta_{p}\right)^{-m} u-\left(\lambda-\frac{1}{p} \Delta_{p}^{X_{0}}\right)^{-m} \psi u \\
& \quad=\left(\lambda-\frac{1}{p} \Delta_{p}^{X_{0}}\right)^{-m}\left[\left(\lambda-\frac{1}{p} \Delta_{p}^{X_{0}}\right)^{m}, \psi\right]\left(\lambda-\frac{1}{p} \Delta_{p}\right)^{-m} u
\end{aligned}
$$

Now, for any $p \in \mathbb{N}^{*}, \lambda \in \delta$ and $W \in X_{0}$, by Theorem 4.2 and (4.13), we have for $m>2 n+1, v \in\left(L_{0}^{p} \otimes E_{0}\right)_{W},|v|=1$,

$$
\begin{aligned}
\| \phi_{\frac{1}{2} c_{0} \sqrt{p}, W}^{X_{0}}\left(\psi\left(\lambda-\frac{1}{p} \Delta_{p}\right)^{-m}\right. & \left.-\left(\lambda-\frac{1}{p} \Delta_{p}^{X_{0}}\right)^{-m} \psi\right) \delta_{v} \|_{p, n+1} \\
\leq C & \left\|\phi_{\frac{1}{2} c_{0} \sqrt{p}, W}^{X_{0}}\left[\left(\lambda-\frac{1}{p} \Delta_{p}^{X_{0}}\right)^{m}, \psi\right]\left(\lambda-\frac{1}{p} \Delta_{p}\right)^{-m} \delta_{v}\right\|_{p, n+1-m} .
\end{aligned}
$$

Since the operator $\left[\left(\lambda-\frac{1}{p} \Delta_{p}^{X_{0}}\right)^{m}, \psi\right]$ vanishes on $B^{X_{0}}(0,3 \varepsilon)$, for any $W \in B^{X_{0}}(0, \varepsilon)$ we have $d^{X_{0}}(W, Z)>2 \varepsilon$ and, therefore, $\phi_{\frac{1}{2} c_{0} \sqrt{p}, W}^{X_{0}}(Z)=1$ on the support of $\left[\left(\lambda-\frac{1}{p} \Delta_{p}^{X_{0}}\right)^{m}, \psi\right]$. Hence, for $W \in B^{X_{0}}(0, \varepsilon)$, by (4.14), we get

$$
\begin{aligned}
& \left\|\phi_{\frac{1}{2} c_{0} \sqrt{p}, W}^{X_{0}}\left(\psi\left(\lambda-\frac{1}{p} \Delta_{p}\right)^{-m}-\left(\lambda-\frac{1}{p} \Delta_{p}^{X_{0}}\right)^{-m} \psi\right) \delta_{v}\right\|_{p, n+1} \\
& \leq C\left\|\left[\left(\lambda-\frac{1}{p} \Delta_{p}^{X_{0}}\right)^{m}, \psi\right]\left(\lambda-\frac{1}{p} \Delta_{p}\right)^{-m} \delta_{v}\right\|_{p, n+1-m} \\
& \leq C\left\|\left(\lambda-\frac{1}{p} \Delta_{p}\right)^{-m} \delta_{v}\right\|_{p, n+1} \leq C\left\|\delta_{v}\right\|_{p, n+1-m} \leq C p^{n / 2} .
\end{aligned}
$$


and, finally, by (4.10) and (4.15), we get

$$
\sup _{Z, W \in B^{X_{0}(0, \varepsilon / 2)}}\left|R_{\lambda, p}^{(m)}(Z, W)-R_{\lambda, p}^{X_{0},(m)}(Z, W)\right| \leq C_{1} p^{n} e^{-\frac{1}{2} c_{0} \sqrt{p}} \leq C_{2} e^{-\frac{1}{4} c_{0} \sqrt{p}} .
$$

Using (3.58), we complete the proof for $k=0$. The proof of the case of arbitrary $k$ can be given similarly to the proof of Theorem 3.6 .

Now we can proceed as in [15, 23]. We only observe that all the constants in the estimates in [15, 23] depend on finitely many derivatives of $g^{T X}, h^{L}, \nabla^{L}, h^{E}, \nabla^{E}, J$ and the lower bound of $g^{T X}$. Therefore, by the bounded geometry assumptions, all the estimates are uniform on the parameter $x_{0} \in X$. We will omit the details and give only the final result, stating the full off-diagonal asymptotic expansion of the generalized Bergman kernel $P_{q, p}$ as $p \rightarrow \infty$ (see Theorem 4.3 below).

The almost complex structure $J_{x_{0}}$ induces a decomposition $T_{x_{0}} X \otimes_{\mathbb{R}} \mathbb{C}=T_{x_{0}}^{(1,0)} X \oplus T_{x_{0}}^{(0,1)} X$, where $T_{x_{0}}^{(1,0)} X$ and $T_{x_{0}}^{(0,1)} X$ are the eigenspaces of $J_{x_{0}}$ corresponding to eigenvalues $i$ and $-i$ respectively. Denote by $\operatorname{det}_{\mathbb{C}}$ the determinant function of the complex space $T_{x_{0}}^{(1,0)} X$. Put

$$
\mathcal{J}_{x_{0}}=-2 \pi i J_{0}
$$

Then $\mathcal{J}_{x_{0}}: T_{x_{0}}^{(1,0)} X \rightarrow T_{x_{0}}^{(1,0)} X$ is positive, and $\mathcal{J}_{x_{0}}: T_{x_{0}} X \rightarrow T_{x_{0}} X$ is skew-adjoint [23, (1.81)] (cf. [10, (4.114)]). We define a function $\mathcal{P}=\mathcal{P}_{x_{0}} \in \mathscr{C}^{\infty}\left(T_{x_{0}} X \times T_{x_{0}} X\right)$ by

$$
\mathcal{P}\left(Z, Z^{\prime}\right)=\frac{\operatorname{det}_{\mathbb{C}} \mathcal{J}_{x_{0}}}{(2 \pi)^{n}} \exp \left(-\frac{1}{4}\left\langle\left(\mathcal{J}_{x_{0}}^{2}\right)^{1 / 2}\left(Z-Z^{\prime}\right),\left(Z-Z^{\prime}\right)\right\rangle+\frac{1}{2}\left\langle\mathcal{J}_{x_{0}} Z, Z^{\prime}\right\rangle\right) .
$$

It is the Bergman kernel of the second order differential operator $\mathcal{L}_{0}$ on $\mathscr{C}^{\infty}\left(T_{x_{0}} X, \mathbb{C}\right)$ given by

$$
\mathcal{L}_{0}=-\sum_{j=1}^{2 n}\left(\nabla_{e_{j}}+\frac{1}{2} R_{x_{0}}^{L}\left(Z, e_{j}\right)\right)^{2}-\tau\left(x_{0}\right),
$$

where $\left\{e_{j}\right\}_{j=1, \ldots, 2 n}$ is an orthonormal base in $T_{x_{0}} X$. Here, for $U \in T_{x_{0}} X$, we denote by $\nabla_{U}$ the ordinary operator of differentiation in the direction $U$ on the space $\mathscr{C}^{\infty}\left(T_{x_{0}} X, \mathbb{C}\right)$. Thus, $\mathcal{P}$ is the smooth kernel (with respect to $d v_{T X}$ ) of the orthogonal projection in $L^{2}\left(T_{x_{0}} X, \mathbb{C}\right)$ to the kernel of $\mathcal{L}_{0}$.

Let $\kappa$ be the function defined in (4.3).

Theorem 4.3. There exists $\varepsilon \in\left(0, a^{X}\right)$ such that, for any $j, m, m^{\prime} \in \mathbb{N}, j \geq 2 q$, there exist positive constants $C$, $c$ and $M$ such that for any $p \geq 1, x_{0} \in X$ and $Z, Z^{\prime} \in T_{x_{0}} X,|Z|,\left|Z^{\prime}\right|<\varepsilon$, we have

$$
\begin{gathered}
\sup _{|\alpha|+\left|\alpha^{\prime}\right| \leq m}\left|\frac{\partial^{|\alpha|+\left|\alpha^{\prime}\right|}}{\partial Z^{\alpha} \partial Z^{\prime \alpha^{\prime}}}\left(\frac{1}{p^{n}} P_{q, p, x_{0}}\left(Z, Z^{\prime}\right)-\sum_{r=2 q}^{j} F_{q, r, x_{0}}\left(\sqrt{p} Z, \sqrt{p} Z^{\prime}\right) \kappa^{-\frac{1}{2}}(Z) \kappa^{-\frac{1}{2}}\left(Z^{\prime}\right) p^{-\frac{r}{2}+q}\right)\right|_{\mathscr{C} m^{\prime}(X)} \\
\leq C p^{-\frac{j-m+1}{2}+q}\left(1+\sqrt{p}|Z|+\sqrt{p}\left|Z^{\prime}\right|\right)^{M} \exp \left(-c \sqrt{\mu_{0} p}\left|Z-Z^{\prime}\right|\right)+\mathcal{O}\left(e^{-c_{0} \sqrt{p}}\right),
\end{gathered}
$$

where

$$
F_{q, r, x_{0}}\left(Z, Z^{\prime}\right)=J_{q, r, x_{0}}\left(Z, Z^{\prime}\right) \mathcal{P}_{x_{0}}\left(Z, Z^{\prime}\right)
$$


$J_{q, r, x_{0}}\left(Z, Z^{\prime}\right)$ are polynomials in $Z, Z^{\prime}$, depending smoothly on $x_{0}$, with the same parity as $r$ and $\operatorname{deg} J_{q, r, x_{0}} \leq 3 r$.

Here $\mathscr{C}^{m^{\prime}}(X)$ is the $\mathscr{C}^{m^{\prime}}$-norm for the parameter $x_{0} \in X$. Note that the summation in (4.20) starts from $r=2 q$ and (4.21) due to [23, Th 1.18].

\section{BEREZIN-TOEPLITZ QUANTIZATION ON ORBIFOLDS}

After the pioneering work of Berezin, the Berezin-Toeplitz quantization achieved generality for compact Kähler manifolds and trivial bundle $E$ through the works [6, 7, 4]. We refer to [20, 22, 23, 25] for more references and background. The theory of Berezin-Toeplitz quantization on Kähler and symplectic orbifolds was first established by Ma and Marinescu [24, Theorems $6.14,6.16]$ by using as quantum spaces the kernel of the $\operatorname{spin}^{c}$ Dirac operator. Especially, they showed that the set of Toeplitz operators forms an algebra. The main tool was the asymptotic expansion of the Bergman kernel associated with the $\operatorname{spin}^{c}$ Dirac operator of Dai-Liu-Ma [10].

In this Section, we establish the corresponding theory for the renormalized Bochner-Laplacian on symplectic orbifolds. In [22, $§ 5.4]$ one can find more explanations and references for Sections 5.1 and 5.2. For related topics about orbifolds we refer to [1].

This Section is organized as follows. In Section 5.1 we recall the basic definitions about orbifolds. In Section 5.2 we explain the asymptotic expansion of Bergman kernel on symplectic orbifolds, which we apply in Section 5.3 to derive the Berezin-Toeplitz quantization on symplectic orbifolds.

5.1. Basic definitions on orbifolds. We define at first a category $\mathcal{M}_{s}$ as follows : The objects of $\mathcal{M}_{s}$ are the class of pairs $(G, M)$ where $M$ is a connected smooth manifold and $G$ is a finite group acting effectively on $M$ (i.e., if $g \in G$ such that $g x=x$ for any $x \in M$, then $g$ is the unit element of $G$ ). If $(G, M)$ and $\left(G^{\prime}, M^{\prime}\right)$ are two objects, then a morphism $\Phi:(G, M) \rightarrow\left(G^{\prime}, M^{\prime}\right)$ is a family of open embeddings $\varphi: M \rightarrow M^{\prime}$ satisfying :

i) For each $\varphi \in \Phi$, there is an injective group homomorphism $\lambda_{\varphi}: G \rightarrow G^{\prime}$ that makes $\varphi$ be $\lambda_{\varphi}$-equivariant.

ii) For $g \in G^{\prime}, \varphi \in \Phi$, we define $g \varphi: M \rightarrow M^{\prime}$ by $(g \varphi)(x)=g \varphi(x)$ for $x \in M$. If $(g \varphi)(M) \cap \varphi(M) \neq \emptyset$, then $g \in \lambda_{\varphi}(G)$.

iii) For $\varphi \in \Phi$, we have $\Phi=\left\{g \varphi: g \in G^{\prime}\right\}$.

Definition 5.1. Let $X$ be a paracompact Hausdorff space. A $m$-dimensional orbifold chart on $X$ consists of a connected open set $U$ of $X$, an object $\left(G_{U}, \widetilde{U}\right)$ of $\mathcal{M}_{s}$ with $\operatorname{dim} \widetilde{U}=m$, and a ramified covering $\tau_{U}: \widetilde{U} \rightarrow U$ which is $G_{U}$-invariant and induces a homeomorphism $U \simeq \widetilde{U} / G_{U}$. We denote the chart by $\left(G_{U}, \widetilde{U}\right) \stackrel{\tau_{U}}{\longrightarrow} U$.

A $m$-dimensional orbifold atlas $\mathcal{V}$ on $X$ consists of a family of $m$-dimensional orbifold charts $\mathcal{V}(U)=\left(\left(G_{U}, \widetilde{U}\right) \stackrel{\tau_{U}}{\longrightarrow} U\right)$ satisfying the following conditions :

i) The open sets $U \subset X$ form a covering $\mathcal{U}$ with the property:

(5.1) For any $U, U^{\prime} \in \mathcal{U}$ and $x \in U \cap U^{\prime}$, there is $U^{\prime \prime} \in \mathcal{U}$ such that $x \in U^{\prime \prime} \subset U \cap U^{\prime}$.

ii) for any $U, V \in \mathcal{U}, U \subset V$ there exists a morphism $\varphi_{V U}:\left(G_{U}, \widetilde{U}\right) \rightarrow\left(G_{V}, \widetilde{V}\right)$, which covers the inclusion $U \subset V$ and satisfies $\varphi_{W U}=\varphi_{W V} \circ \varphi_{V U}$ for any $U, V, W \in \mathcal{U}$, with $U \subset V \subset W$. 
It is easy to see that there exists a unique maximal orbifold atlas $\mathcal{V}_{\max }$ containing $\mathcal{V}$; $\mathcal{V}_{\max }$ consists of all orbifold charts $\left(G_{U}, \widetilde{U}\right) \stackrel{\tau_{U}}{\longrightarrow} U$, which are locally isomorphic to charts from $\mathcal{V}$ in the neighborhood of each point of $U$. A maximal orbifold atlas $\mathcal{V}_{\text {max }}$ is called an orbifold structure and the pair $\left(X, \mathcal{V}_{\max }\right)$ is called an orbifold. As usual, once we have an orbifold atlas $\mathcal{V}$ on $X$ we denote the orbifold by $(X, \mathcal{V})$, since $\mathcal{V}$ determines uniquely $\mathcal{V}_{\max }$.

Note that if $\mathcal{U}^{\prime}$ is a refinement of $\mathcal{U}$ satisfying (5.1), then there is an orbifold atlas $\mathcal{V}^{\prime}$ such that $\mathcal{V} \cup \mathcal{V}^{\prime}$ is an orbifold atlas, hence $\mathcal{V} \cup \mathcal{V}^{\prime} \subset \mathcal{V}_{\text {max }}$. This shows that we may choose $\mathcal{U}$ arbitrarily fine.

Let $(X, \mathcal{V})$ be an orbifold. For each $x \in X$, we can choose a small neighborhood $\left(G_{x}, \widetilde{U}_{x}\right) \rightarrow$ $U_{x}$ such that $x \in \widetilde{U}_{x}$ is a fixed point of $G_{x}$ (it follows from the definition that such a $G_{x}$ is unique up to isomorphisms for each $x \in X$ ). We denote by $\left|G_{x}\right|$ the cardinal of $G_{x}$. If $\left|G_{x}\right|=1$, then $X$ has a smooth manifold structure in the neighborhood of $x$, which is called a smooth point of $X$. If $\left|G_{x}\right|>1$, then $X$ is not a smooth manifold in the neighborhood of $x$, which is called a singular point of $X$. We denote by $X_{\text {sing }}=\left\{x \in X ;\left|G_{x}\right|>1\right\}$ the singular set of $X$, and $X_{\text {reg }}=\left\{x \in X ;\left|G_{x}\right|=1\right\}$ the regular set of $X$.

It is useful to note that on an orbifold $(X, \mathcal{V})$ we can construct partitions of unity. First, let us call a function on $X$ smooth, if its lift to any chart of the orbifold atlas $\mathcal{V}$ is smooth in the usual sense. Then the definition and construction of a smooth partition of unity associated to a locally finite covering carries over easily from the manifold case. The point is to construct smooth $G_{U}$-invariant functions with compact support on $\left(G_{U}, \widetilde{U}\right)$.

In Definition 5.1 we can replace $\mathcal{M}_{s}$ by a category of manifolds with an additional structure such as orientation, Riemannian metric, almost-complex structure or complex structure. We impose that the morphisms (and the groups) preserve the specified structure. So we can define oriented, Riemannian, almost-complex or complex orbifolds.

Let $(X, \mathcal{V})$ be an arbitrary orbifold. By the above definition, a Riemannian metric on $X$ is a Riemannian metric $g^{T X}$ on $X_{\text {reg }}$ such that the lift of $g^{T X}$ to any chart of the orbifold atlas $\mathcal{V}$ can be extended to a smooth Riemannian metric. Certainly, for any $\left(G_{U}, \widetilde{U}\right) \in \mathcal{V}$, we can always construct a $G_{U}$-invariant Riemannian metric on $\widetilde{U}$. By a partition of unity argument, we see that there exist Riemannian metrics on the orbifold $(X, \mathcal{V})$.

Definition 5.2. An orbifold vector bundle $E$ over an orbifold $(X, \mathcal{V})$ is defined as follows : $E$ is an orbifold and for $U \in \mathcal{U},\left(G_{U}^{E}, \widetilde{p}_{U}: \widetilde{E}_{U} \rightarrow \widetilde{U}\right)$ is a $G_{U}^{E}$-equivariant vector bundle, $\left(G_{U}^{E}, \widetilde{E}_{U}\right)$ (resp. $\left(G_{U}=G_{U}^{E} / K_{U}^{E}, \widetilde{U}\right), K_{U}^{E}=\operatorname{Ker}\left(G_{U}^{E} \rightarrow \operatorname{Diffeo}(\widetilde{U})\right)$ ) is the orbifold structure of $E$ (resp. $X)$ and morphisms for $\left(G_{U}^{E}, \widetilde{E}_{U}\right)$ are morphisms of equivariant vector bundles. If $G_{U}^{E}$ acts effectively on $\widetilde{U}$ for $U \in \mathcal{U}$, i.e., $K_{U}^{E}=\{1\}$, we call $E$ a proper orbifold vector bundle.

Note that any structure on $X$ or $E$ is locally $G_{x}$ or $G_{U_{x}}^{E}$-equivariant.

Remark 5.3. Let $E$ be an orbifold vector bundle on $(X, \mathcal{V})$. For $U \in \mathcal{U}$, let $\widetilde{E_{U}^{\mathrm{pr}}}$ be the maximal $K_{U}^{E}$-invariant sub-bundle of $\widetilde{E}_{U}$ on $\widetilde{U}$. Then $\left(G_{U}, \widetilde{E_{U}^{\mathrm{pr}}}\right)$ defines a proper orbifold vector bundle on $(X, \mathcal{V})$, denoted by $E^{\mathrm{pr}}$.

The (proper) orbifold tangent bundle $T X$ on an orbifold $X$ is defined by $\left(G_{U}, T \widetilde{U} \rightarrow \widetilde{U}\right)$, for $U \in \mathcal{U}$. In the same vein we introduce the cotangent bundle $T^{*} X$. We can form tensor products of bundles by taking the tensor products of their local expressions in the charts of 
an orbifold atlas. Note that a Riemannian metric on $X$ induces a section of $T^{*} X \otimes T^{*} X$ over $X$ which is a positive definite bilinear form on $T_{x} X$ at each point $x \in X$.

Let $E \rightarrow X$ be an orbifold vector bundle and $k \in \mathbb{N} \cup\{\infty\}$. A section $s: X \rightarrow E$ is called $\mathscr{C}^{k}$ if for each $U \in \mathcal{U},\left.s\right|_{U}$ is covered by a $G_{U}^{E}$-invariant $\mathscr{C}^{k}$ section $\widetilde{s}_{U}: \widetilde{U} \rightarrow \widetilde{E}_{U}$. We denote by $\mathscr{C}^{k}(X, E)$ the space of $\mathscr{C}^{k}$ sections of $E$ on $X$.

If $X$ is oriented, we define the integral $\int_{X} \alpha$ for a form $\alpha$ over $X$ (i.e., a section of $\Lambda\left(T^{*} X\right)$ over $X$ ) as follows. If $\operatorname{supp}(\alpha) \subset U \in \mathcal{U}$, set

$$
\int_{X} \alpha:=\frac{1}{\left|G_{U}\right|} \int_{\widetilde{U}} \widetilde{\alpha}_{U}
$$

It is easy to see that the definition is independent of the chart. For general $\alpha$ we extend the definition by using a partition of unity.

If $X$ is an oriented Riemannian orbifold, there exists a canonical volume element $d v_{X}$ on $X$, which is a section of $\Lambda^{m}\left(T^{*} X\right), m=\operatorname{dim} X$. Hence, we can also integrate functions on $X$.

Assume now that the Riemannian orbifold $(X, \mathcal{V})$ is compact. For $x, y \in X$, put

$$
\begin{aligned}
d(x, y)= & \operatorname{Inf}_{\gamma}\left\{\sum_{i} \int_{t_{i-1}}^{t_{i}}\left|\frac{\partial}{\partial t} \widetilde{\gamma}_{i}(t)\right| d t \mid \gamma:[0,1] \rightarrow X, \gamma(0)=x, \gamma(1)=y,\right. \\
& \text { such that there exist } t_{0}=0<t_{1}<\cdots<t_{k}=1, \gamma\left(\left[t_{i-1}, t_{i}\right]\right) \subset U_{i}, \\
& \left.U_{i} \in \mathcal{U}, \text { and a } \mathscr{C}^{\infty} \operatorname{map} \widetilde{\gamma}_{i}:\left[t_{i-1}, t_{i}\right] \rightarrow \widetilde{U}_{i} \text { that covers }\left.\gamma\right|_{\left[t_{i-1}, t_{i}\right]}\right\} .
\end{aligned}
$$

Then $(X, d)$ is a metric space.

Let us discuss briefly kernels and operators on orbifolds. For any open set $U \subset X$ and orbifold chart $\left(G_{U}, \widetilde{U}\right) \stackrel{\tau_{U}}{\longrightarrow} U$, we will add a superscript ${ }^{\sim}$ to indicate the corresponding objects on $\widetilde{U}$. Assume that $\widetilde{\mathcal{K}}\left(\widetilde{x}, \widetilde{x}^{\prime}\right) \in \mathscr{C}^{\infty}\left(\widetilde{U} \times \widetilde{U}, \pi_{1}^{*} \widetilde{E} \otimes \pi_{2}^{*} \widetilde{E}^{*}\right)$ verifies

$$
(g, 1) \widetilde{\mathcal{K}}\left(g^{-1} \widetilde{x}, \widetilde{x}^{\prime}\right)=\left(1, g^{-1}\right) \widetilde{\mathcal{K}}\left(\widetilde{x}, g \widetilde{x}^{\prime}\right) \quad \text { for any } g \in G_{U},
$$

where $\left(g_{1}, g_{2}\right)$ acts on $\widetilde{E}_{\widetilde{x}} \times \widetilde{E}_{\widetilde{x}}^{*}$ by $\left(g_{1}, g_{2}\right)\left(\xi_{1}, \xi_{2}\right)=\left(g_{1} \xi_{1}, g_{2} \xi_{2}\right)$.

We define the operator $\widetilde{\mathcal{K}}: \mathscr{C}_{0}^{\infty}(\widetilde{U}, \widetilde{E}) \rightarrow \mathscr{C}^{\infty}(\widetilde{U}, \widetilde{E})$ by

$$
(\widetilde{\mathcal{K}} \widetilde{s})(\widetilde{x})=\int_{\widetilde{U}} \widetilde{\mathcal{K}}\left(\widetilde{x}, \widetilde{x}^{\prime}\right) \widetilde{s}\left(\widetilde{x}^{\prime}\right) d v_{\widetilde{U}}\left(\widetilde{x}^{\prime}\right) \quad \text { for } \widetilde{s} \in \mathscr{C}_{0}^{\infty}(\widetilde{U}, \widetilde{E}) .
$$

Any element $g \in G_{U}$ acts on $\mathscr{C}^{\infty}(\widetilde{U}, \widetilde{E})$ by: $(g \cdot \widetilde{s})(\widetilde{x}):=g \cdot \widetilde{s}\left(g^{-1} \widetilde{x}\right)$, where $\widetilde{s} \in \mathscr{C}^{\infty}(\widetilde{U}, \widetilde{E})$. We can then identify an element $s \in \mathscr{C}^{\infty}(U, E)$ with an element $\widetilde{s} \in \mathscr{C}^{\infty}(\widetilde{U}, \widetilde{E})$ verifying $g \cdot \widetilde{s}=\widetilde{s}$ for any $g \in G_{U}$.

With this identification, we define the operator $\mathcal{K}: \mathscr{C}_{0}^{\infty}(U, E) \rightarrow \mathscr{C}^{\infty}(U, E)$ by

$$
(\mathcal{K} s)(x)=\frac{1}{\left|G_{U}\right|} \int_{\widetilde{U}} \widetilde{\mathcal{K}}\left(\widetilde{x}, \widetilde{x}^{\prime}\right) \widetilde{s}\left(\widetilde{x}^{\prime}\right) d v_{\widetilde{U}}\left(\widetilde{x}^{\prime}\right) \quad \text { for } s \in \mathscr{C}_{0}^{\infty}(U, E),
$$

where $\widetilde{x} \in \tau_{U}^{-1}(x)$. Then the smooth kernel $\mathcal{K}\left(x, x^{\prime}\right)$ of the operator $\mathcal{K}$ with respect to $d v_{X}$ is (cf. [10, (5.18)])

$$
\mathcal{K}\left(x, x^{\prime}\right)=\sum_{g \in G_{U}}(g, 1) \widetilde{\mathcal{K}}\left(g^{-1} \widetilde{x}, \widetilde{x}^{\prime}\right)
$$


Let $\mathcal{K}_{1}, \mathcal{K}_{2}$ be two operators as above and assume that the kernel of one of $\widetilde{\mathcal{K}}_{1}, \widetilde{\mathcal{K}}_{2}$ has compact support. By (5.2), (5.3), and (5.5), the kernel of $\mathcal{K}_{1} \circ \mathcal{K}_{2}$ is given by

$$
\left(\mathcal{K}_{1} \circ \mathcal{K}_{2}\right)\left(x, x^{\prime}\right)=\sum_{g \in G_{U}}(g, 1)\left(\widetilde{\mathcal{K}}_{1} \circ \widetilde{\mathcal{K}}_{2}\right)\left(g^{-1} \widetilde{x}, \widetilde{x}^{\prime}\right) .
$$

5.2. Bergman kernel on symplectic orbifolds. Let $(X, \omega)$ be a compact symplectic orbifold of dimension $2 n$. Assume that there exists a proper orbifold Hermitian line bundle $\left(L, h^{L}\right)$ on $X$ with a Hermitian connection $\nabla^{L}: \mathscr{C}^{\infty}(X, L) \rightarrow \mathscr{C}^{\infty}\left(X, T^{*} X \otimes L\right)$ satisfying the prequantization condition:

$$
\frac{i}{2 \pi} R^{L}=\omega
$$

where $R^{L}=\left(\nabla^{L}\right)^{2}$ is the curvature of $\nabla^{L}$. Let $\left(E, h^{E}\right)$ be a proper orbifold Hermitian vector bundle on $X$ equipped with a Hermitian connection $\nabla^{E}$ and $R^{E}$ be the curvature of $\nabla^{E}$.

Let $g^{T X}$ be a Riemannian metric on $X$. Let $\Delta_{p}$ be the renormalized Bochner-Laplacian acting on $\mathscr{C}^{\infty}\left(X, L^{p} \otimes E\right)$ by (1.5). With the same proof as in [21, Corollary 1.2], we can establish the spectral gap property.

Theorem 5.4. Let $(X, \omega)$ be a compact symplectic orbifold, $\left(L, \nabla^{L}, h^{L}\right)$ be a prequantum Hermitian proper orbifold line bundle on $(X, \omega)$ and $\left(E, \nabla^{E}, h^{E}\right)$ be an arbitrary Hermitian proper orbifold vector bundle on $X$. There exists $C_{L}>0$ such that for any $p$

$$
\sigma\left(\Delta_{p}\right) \subset\left[-C_{L}, C_{L}\right] \cup\left[2 p \mu_{0}-C_{L},+\infty\right),
$$

where $\mu_{0}>0$ is given by (1.7).

From now on, we assume $p>C_{L}\left(2 \mu_{0}\right)^{-1}$. We consider the subspace $\mathcal{H}_{p} \subset L^{2}\left(X, L^{p} \otimes E\right)$ spanned by the eigensections of $\Delta_{p}$ corresponding to eigenvalues in $\left[-C_{L}, C_{L}\right]$. We define the generalized Bergman kernel

$$
P_{p}(\cdot, \cdot) \in \mathscr{C}^{\infty}\left(X \times X, \pi_{1}^{*}\left(L^{p} \otimes E\right) \otimes \pi_{2}^{*}\left(\left(L^{p} \otimes E\right)^{*}\right)\right)
$$

as the smooth kernel with respect to the Riemannian volume form $d v_{X}\left(x^{\prime}\right)$ of the orthogonal projection (Bergman projection) $P_{\mathcal{H}_{p}}$ from $L^{2}\left(X, L^{p} \otimes E\right)$ onto $\mathcal{H}_{p}$.

Consider an open set $U \subset X$ and orbifold chart $\left(G_{U}, \widetilde{U}\right) \stackrel{\tau_{U}}{\longrightarrow} U$. Recall that we add a superscript ${ }^{\sim}$ to indicate the corresponding objects on $\widetilde{U}$. The Riemannian metric $g^{T X}$ can be lifted to a $G_{U}$-invariant Riemannian metric $g^{\widetilde{U}}$ on $\widetilde{U}$. We denote by $B^{\widetilde{U}}(\widetilde{x}, \varepsilon)$ and $B^{T_{\widetilde{x}}} \widetilde{U}(0, \varepsilon)$ the open balls in $\widetilde{U}$ and $T_{\widetilde{x}} \widetilde{U}$ with center $\widetilde{x}$ and 0 and radius $\varepsilon$, respectively. We will always assume that $\tau_{U}$ extends to $\left(G_{U}, \widetilde{V}\right) \stackrel{\tau_{V}}{\longrightarrow} V$ with $U \subset \subset V$ and $\widetilde{U} \subset \subset \widetilde{V}$. Let $\partial U=\bar{U} \backslash U$ and $\partial \widetilde{U}=\overline{\widetilde{U}} \backslash \widetilde{U}$. Fix $a^{X}>0$ such that for every open set $U \subset X$ and orbifold chart $\left(G_{U}, \widetilde{U}\right) \stackrel{\tau_{U}}{\longrightarrow} U$, for every $\varepsilon \leq a^{X}$ and for every $\widetilde{x} \in \widetilde{U}$ such that $d(\widetilde{x}, \partial \widetilde{U}) \leq \varepsilon$, the exponential map $T_{\widetilde{x}} \widetilde{U}$ $Z \mapsto \exp _{\widetilde{x}}^{X}(Z) \in \widetilde{U}$ is a diffeomorphism from $B^{T \widetilde{x}}(0, \varepsilon)$ onto $B^{\widetilde{U}}(\widetilde{x}, \varepsilon)$. Throughout in what follows, $\varepsilon$ runs in the fixed interval $\left(0, a^{X} / 4\right)$.

Let $f: \mathbb{R} \rightarrow[0,1]$ be a smooth even function such that $f(v)=1$ for $|v| \leqslant \varepsilon / 2$, and $f(v)=0$ for $|v| \geqslant \varepsilon$. Set

$$
F(a)=\left(\int_{-\infty}^{+\infty} f(v) d v\right)^{-1} \int_{-\infty}^{+\infty} e^{i v a} f(v) d v
$$


Then $F(a)$ is an even function and lies in the Schwartz space $\mathcal{S}(\mathbb{R})$ and $F(0)=1$. Let $\widetilde{F}$ be the holomorphic function on $\mathbb{C}$ such that $\widetilde{F}\left(a^{2}\right)=F(a)$. The restriction of $\widetilde{F}$ to $\mathbb{R}$ lies in the Schwartz space $\mathcal{S}(\mathbb{R})$. Then there exists $\left\{c_{j}\right\}_{j=1}^{\infty}$ such that for any $k \in \mathbb{N}$, the function

$$
\widetilde{F}_{k}(a)=\widetilde{F}(a)-\sum_{j=1}^{k} c_{j} a^{j} \widetilde{F}(a), \quad c_{k+1}=\frac{1}{(k+1) !} \widetilde{F}_{k}^{(k+1)}(0),
$$

verifies

$$
\widetilde{F}_{k}^{(i)}(0)=0 \text { for any } 0<i \leqslant k .
$$

Using the same arguments as in [23], one can show the analog of [23, Proposition 1.2]:

Proposition 5.5. For any $k, m \in \mathbb{N}$, there exists $C_{k, m}>0$ such that for $p \geqslant 1$

$$
\left|\widetilde{F}_{k}\left(\frac{1}{\sqrt{p}} \Delta_{p}\right)\left(x, x^{\prime}\right)-P_{p}\left(x, x^{\prime}\right)\right|_{\mathscr{C}^{m}(X \times X)} \leqslant C_{k, m} p^{-\frac{k}{2}+2(2 m+2 n+1)} .
$$

Here the $\mathscr{C}^{m}$ norm is induced by $\nabla^{L}, \nabla^{E}$ and $h^{L}, h^{E}, g^{T X}$.

Using (5.11), (5.12), and the property of the finite propagation speed of solutions of hyperbolic equations [22, Appendix D.2] (which still holds on orbifolds as pointed out in [19]) it is clear that for $x, x^{\prime} \in X, \widetilde{F}_{k}\left(\frac{1}{\sqrt{p}} \Delta_{p}\right)(x, \cdot)$ only depends on the restriction of $\Delta_{p}$ to $B^{X}\left(x, \varepsilon p^{-\frac{1}{4}}\right)$, and $\widetilde{F}_{k}\left(\frac{1}{\sqrt{p}} \Delta_{p}\right)\left(x, x^{\prime}\right)=0$, if $d\left(x, x^{\prime}\right) \geqslant \varepsilon p^{-\frac{1}{4}}$.

Consider an open set $U \subset X$ with an orbifold chart $\left(G_{U}, \widetilde{U}\right) \stackrel{\tau_{U}}{\longrightarrow} U$. Let

$$
U_{1}=\{x \in U, d(x, \partial U)<2 \varepsilon\}
$$

For any $x_{0} \in U_{1}$, the exponential map $\exp _{\widetilde{x}_{0}}$ is a diffeomorphism from $B^{T_{x_{0}} \widetilde{U_{1}}}(0,2 \varepsilon)$ onto $B^{\widetilde{U_{1}}}\left(\widetilde{x}_{0}, 2 \varepsilon\right)$ which is $G_{x_{0}}$-equivariant. Thus we can extend everything from $B^{T_{x_{0}} \widetilde{U_{1}}}(0,2 \varepsilon)$ to $\widetilde{X_{0}}:=T_{\widetilde{x}_{0}} \widetilde{U_{1}}$ as in Section 4 (cf. [23, $\left.\S 1.2\right]$ ) which is automatically $G_{x_{0}}$-equivariant. Let $P_{\widetilde{X_{0}}, p}$ be the spectral projection of the renormalized Bochner-Laplacian $\Delta_{p}^{\widetilde{X_{0}}}$ on $\mathscr{C}^{\infty}\left(\widetilde{X_{0}}, L_{0}^{p} \otimes E_{0}\right)$, corresponding to the interval $\left[-C_{L_{0}}, C_{L_{0}}\right]$, and $P_{\widetilde{X}_{0}, p}(\widetilde{x}, \widetilde{y})$ be the Schwartz kernel of $P_{\widetilde{X}_{0}, p}$ with respect to the volume form $d v_{\widetilde{X_{0}}}$. Let $P_{X_{0}, p}(x, y)$ be the corresponding object on $G_{x_{0}} \backslash T_{\widetilde{x}_{0}} \widetilde{U_{1}}$, then, by (5.6), we have

$$
P_{X_{0}, p}(x, y)=\sum_{g \in G_{x_{0}}}(g, 1) P_{\widetilde{X}_{0}, p}\left(g^{-1} \widetilde{x}, \widetilde{y}^{\prime}\right) .
$$

By Proposition 5.5, we get the analog of [23, Proposition 1.3]: for any $\ell, m \in \mathbb{N}$, there exists $C_{\ell, m}>0$ such that, for any $x, y \in B\left(x_{0}, \varepsilon\right)$ and $p \in \mathbb{N}^{*}$

$$
\left|P_{p}(x, y)-P_{X_{0}, p}(x, y)\right| \leq C_{\ell, m} p^{-\ell}
$$

5.3. Berezin-Toeplitz quantization on symplectic orbifolds. We apply now the results of Section 5.2 to establish the Berezin-Toeplitz quantization on symplectic orbifolds by using as quantum spaces the spaces $\mathcal{H}_{p}$. We use the notations and assumptions of that Section. We will closely follow and slightly modify the arguments of [24, $\S 6.3,6.4]$.

Thus we have the following definition. 
Definition 5.6. A Toeplitz operator is a sequence $\left\{T_{p}\right\}_{p \in \mathbb{N}}$ of bounded linear operators

$$
T_{p}: L^{2}\left(X, L^{p} \otimes E\right) \longrightarrow L^{2}\left(X, L^{p} \otimes E\right)
$$

satisfying the following conditions:

(i): For any $p \in \mathbb{N}$, we have

$$
T_{p}=P_{\mathcal{H}_{p}} T_{p} P_{\mathcal{H}_{p}}
$$

(ii): There exists a sequence $g_{l} \in \mathscr{C}^{\infty}(X, \operatorname{End}(E))$ such that

$$
T_{p}=P_{\mathcal{H}_{p}}\left(\sum_{l=0}^{\infty} p^{-l} g_{l}\right) P_{\mathcal{H}_{p}}+\mathcal{O}\left(p^{-\infty}\right),
$$

that is, for any $k \geq 0$ there exists $C_{k}>0$ such that

$$
\left\|T_{p}-P_{\mathcal{H}_{p}}\left(\sum_{l=0}^{k} p^{-l} g_{l}\right) P_{\mathcal{H}_{p}}\right\| \leq C_{k} p^{-k-1} .
$$

For any section $f \in \mathscr{C}^{\infty}(X$, End $(E))$, the Berezin-Toeplitz quantization of $f$ is defined by

$$
T_{f, p}: L^{2}\left(X, L^{p} \otimes E\right) \longrightarrow L^{2}\left(X, L^{p} \otimes E\right), \quad T_{f, p}=P_{p} f P_{p} .
$$

The Schwartz kernel of $T_{f, p}$ is given by

$$
T_{f, p}\left(x, x^{\prime}\right)=\int_{X} P_{p}\left(x, x^{\prime \prime}\right) f\left(x^{\prime \prime}\right) P_{p}\left(x^{\prime \prime}, x^{\prime}\right) d v_{X}\left(x^{\prime \prime}\right) .
$$

Lemma 5.7. For any $\varepsilon_{0}>0$ and any $l, m \in \mathbb{N}$ there exists $C_{l, m, \varepsilon}>0$ such that

$$
\left|T_{f, p}\left(x, x^{\prime}\right)\right|_{\mathscr{C}^{m}(X \times X)} \leqslant C_{l, m, \varepsilon} p^{-l}
$$

for all $p \geqslant 1$ and all $\left(x, x^{\prime}\right) \in X \times X$ with $d\left(x, x^{\prime}\right)>\varepsilon_{0}$.

Proof. By using Proposition 5.5, the proof is exactly the same as the proof of [24, Lemma 4.2].

Next we obtain the asymptotic expansion of the kernel $T_{f, p}\left(x, x^{\prime}\right)$ in a neighborhood of the diagonal. We will use [24, Condition 6.7].

Recall that the (proper) orbifold tangent bundle $T X$ on an orbifold $X$ is defined by a family of $G_{U}$-equivariant vector bundles $\left(G_{U}, T \widetilde{U} \rightarrow \widetilde{U}\right)$, for $U \in \mathcal{U}$. Consider the fiberwise product $T X \times_{X} T X=\left\{\left(Z, Z^{\prime}\right) \in T_{x_{0}} X \times T_{x_{0}} X: x_{0} \in X\right\}$. Let $\pi: T X \times_{X} T X \rightarrow X$ be the natural projection given by $\pi\left(Z, Z^{\prime}\right)=x_{0}$. We say that $Q_{r, x_{0}} \in \operatorname{End}(E)_{x_{0}}\left[\widetilde{Z}, \widetilde{Z}^{\prime}\right]$, if for any $U \in \mathcal{U}$, it induces a smooth section $Q_{r, x_{0}} \in \operatorname{End}(E)_{x_{0}}\left[\widetilde{Z}, \widetilde{Z}^{\prime}\right]$ of the vector bundle $\pi^{*}(\operatorname{End}(E))$ on $T \widetilde{U} \times_{\widetilde{U}} T \widetilde{U}$ defined for all $\widetilde{x}_{0} \in \widetilde{U}$ and $Z, Z^{\prime} \in T_{\widetilde{x}_{0}} \widetilde{U}$ and polynomial in $Z, Z^{\prime} \in T_{\widetilde{x}_{0}} \widetilde{U}$.

Let $\left\{\Xi_{p}\right\}_{p \in \mathbb{N}}$ be a sequence of linear operators $\Xi_{p}: L^{2}\left(X, L^{p} \otimes E\right) \longrightarrow L^{2}\left(X, L^{p} \otimes E\right)$ with smooth kernel $\Xi_{p}(x, y)$ with respect to $d v_{X}(y)$.

Condition 5.8. Let $k \in \mathbb{N}$. Assume that there exists a family $\left\{Q_{r, x_{0}}\right\}_{0 \leqslant r \leqslant k, x_{0} \in X}$, satisfying the conditions:

(a) $Q_{r, x_{0}} \in \operatorname{End}(E)_{x_{0}}\left[\widetilde{Z}, \widetilde{Z}^{\prime}\right], \widetilde{Z}, \widetilde{Z}^{\prime} \in T_{\widetilde{x}_{0}} X$,

(b) $\left\{Q_{r, x_{0}}\right\}_{x_{0} \in X}$ is smooth with respect to the parameter $x_{0} \in X$, 
and, for every open set $U \in \mathcal{U}$ and every orbifold chart $\left(G_{U}, \widetilde{U}\right) \stackrel{\tau_{U}}{\longrightarrow} U$, a sequence of kernels

$$
\left\{\widetilde{\Xi}_{p, U}\left(\widetilde{x}, \widetilde{x}^{\prime}\right) \in \mathscr{C}^{\infty}\left(\widetilde{U} \times \widetilde{U}, \pi_{1}^{*}\left(\widetilde{L}^{p} \otimes \widetilde{E}\right) \otimes \pi_{2}^{*}\left(\widetilde{L}^{p} \otimes \widetilde{E}\right)^{*}\right)\right\}_{p \in \mathbb{N}}
$$

such that, for every $\varepsilon^{\prime \prime}>0$ and every $\widetilde{x}, \widetilde{x}^{\prime} \in \widetilde{U}$,

$$
\begin{aligned}
& \left.(g, 1) \widetilde{\Xi}_{p, U}\left(g^{-1} \widetilde{x}, \widetilde{x}^{\prime}\right)=\left(1, g^{-1}\right) \widetilde{\Xi}_{p, U}\left(\widetilde{x}, g \widetilde{x}^{\prime}\right) \quad \text { for any } g \in G_{U} \text { (cf. (5.3) }\right), \\
& \widetilde{\Xi}_{p, U}\left(\widetilde{x}, \widetilde{x}^{\prime}\right)=\mathcal{O}\left(p^{-\infty}\right) \quad \text { for } d\left(x, x^{\prime}\right)>\varepsilon^{\prime \prime}, \\
& \Xi_{p}\left(x, x^{\prime}\right)=\sum_{g \in G_{U}}(g, 1) \widetilde{\Xi}_{p, U}\left(g^{-1} \widetilde{x}, \widetilde{x}^{\prime}\right)+\mathcal{O}\left(p^{-\infty}\right),
\end{aligned}
$$

and moreover, for every relatively compact open subset $\widetilde{V} \subset \widetilde{U}$, the following relation is valid for any $\widetilde{x}_{0} \in \widetilde{V}$ :

$$
p^{-n} \widetilde{\Xi}_{p, U, \widetilde{x}_{0}}\left(\widetilde{Z}, \widetilde{Z}^{\prime}\right) \cong \sum_{r=0}^{k}\left(Q_{r, \widetilde{x}_{0}} \mathcal{P}_{\widetilde{x}_{0}}\right)\left(\sqrt{p} \widetilde{Z}, \sqrt{p} \widetilde{Z}^{\prime}\right) p^{-\frac{r}{2}}+\mathcal{O}\left(p^{-\frac{k+1}{2}}\right) .
$$

which means that there exist $\varepsilon^{\prime}>0$ and $C_{0}>0$ with the following property: for any $m \in \mathbb{N}$, there exist $C>0$ and $M>0$ such that for any $\widetilde{x}_{0} \in \widetilde{V}, p \geq 1$ and $\widetilde{Z}, \widetilde{Z}^{\prime} \in T_{\widetilde{x}_{0}} \widetilde{U},|\widetilde{Z}|,\left|\widetilde{Z}^{\prime}\right|<\varepsilon^{\prime}$, we have with $\kappa$ in (4.3),

$$
\begin{aligned}
\left|p^{-n} \widetilde{\Xi}_{p, U, \widetilde{x}_{0}}\left(\widetilde{Z}, \widetilde{Z}^{\prime}\right) \kappa^{\frac{1}{2}}(\widetilde{Z}) \kappa^{\frac{1}{2}}\left(\widetilde{Z}^{\prime}\right)-\sum_{r=0}^{k}\left(Q_{r, \widetilde{x}_{0}} \mathcal{P}_{\widetilde{x}_{0}}\right)\left(\sqrt{p} \widetilde{Z}, \sqrt{p} \widetilde{Z}^{\prime}\right) p^{-\frac{r}{2}}\right|_{\mathscr{C} m(\widetilde{V})} \\
\leq C p^{-\frac{k+1}{2}}\left(1+\sqrt{p}|\widetilde{Z}|+\sqrt{p}\left|\widetilde{Z}^{\prime}\right|\right)^{M} \exp \left(-\sqrt{C_{0} p}\left|\widetilde{Z}-\widetilde{Z^{\prime}}\right|\right)+\mathcal{O}\left(p^{-\infty}\right)
\end{aligned}
$$

Notation 5.9. If the sequence $\left\{\Xi_{p}: L^{2}\left(X, L^{p} \otimes E\right) \longrightarrow L^{2}\left(X, L^{p} \otimes E\right)\right\}_{p \in \mathbb{N}}$ satisfies Condition 5.8, we write

$$
p^{-n} \Xi_{p, x_{0}}\left(Z, Z^{\prime}\right) \cong \sum_{r=0}^{k}\left(Q_{r, x_{0}} \mathcal{P}_{x_{0}}\right)\left(\sqrt{p} Z, \sqrt{p} Z^{\prime}\right) p^{-\frac{r}{2}}+\mathcal{O}\left(p^{-\frac{k+1}{2}}\right) .
$$

As in [24, Lemma 6.9], one can show that the smooth family $Q_{r, x_{0}} \in \operatorname{End}(E)_{x_{0}}\left[\widetilde{Z}, \widetilde{Z}^{\prime}\right]$ in Condition 5.8 is uniquely determined by $\Xi_{p}$.

Theorem 5.10. There exist polynomials $J_{r, x_{0}} \in \operatorname{End}(E)_{x_{0}}\left[\widetilde{Z}, \widetilde{Z}^{\prime}\right]$ such that, for any $k \in \mathbb{N}$, $Z, Z^{\prime} \in T_{x_{0}} X,|Z|,\left|Z^{\prime}\right|<\varepsilon$, we have

$$
p^{-n} P_{p, x_{0}}\left(Z, Z^{\prime}\right) \cong \sum_{r=0}^{k-1}\left(J_{r, x_{0}} \mathcal{P}_{x_{0}}\right)\left(\sqrt{p} Z, \sqrt{p} Z^{\prime}\right) p^{-\frac{r}{2}}+\mathcal{O}\left(p^{-\frac{k}{2}}\right)
$$

in the sense of Notation 5.9

Proof. By Theorem 4.3 for $P_{\widetilde{X_{0}}, p}(\widetilde{x}, \widetilde{y}),(5.14)$ and (5.15), we get Theorem 5.10 as the analog of [24, Lemmas 4.5, 6.10].

From (5.21) and (5.27), we deduce an analog of [24, Lemmas 4.6, 4.7 and 6.10].

Lemma 5.11. Let $f \in \mathscr{C}^{\infty}(X, \operatorname{End}(E))$. There exists a family $\left\{Q_{r, x_{0}}(f)\right\}_{r \in \mathbb{N}, x_{0} \in X}$ such that

(a) $Q_{r, x_{0}}(f) \in \operatorname{End}(E)_{x_{0}}\left[Z, Z^{\prime}\right]$ are polynomials with the same parity as $r$,

(b) $\left\{Q_{r, x_{0}}(f)\right\}_{r \in \mathbb{N}, x_{0} \in X}$ is smooth with respect to $x_{0} \in X$, 
(c) for every $k \in \mathbb{N}, x_{0} \in X, Z, Z^{\prime} \in T_{x_{0}} X,|Z|,\left|Z^{\prime}\right|<\varepsilon / 2$ we have

$$
p^{-n} T_{f, p, x_{0}}\left(Z, Z^{\prime}\right) \cong \sum_{r=0}^{k}\left(Q_{r, x_{0}}(f) \mathcal{P}_{x_{0}}\right)\left(\sqrt{p} Z, \sqrt{p} Z^{\prime}\right) p^{-\frac{r}{2}}+\mathcal{O}\left(p^{-\frac{k+1}{2}}\right),
$$

in the sense of Notation 5.9

Here $Q_{r, x_{0}}(f)$ are expressed by

$$
Q_{r, x_{0}}(f)=\sum_{r_{1}+r_{2}+|\alpha|=r} \mathcal{K}\left[J_{r_{1}, x_{0}}, \frac{\partial^{\alpha} f_{x_{0}}}{\partial Z^{\alpha}}(0) \frac{Z^{\alpha}}{\alpha !} J_{r_{2}, x_{0}}\right] .
$$

Especially,

$$
\begin{gathered}
Q_{0, x_{0}}(f)=f\left(x_{0}\right) . \\
Q_{1, x_{0}}(f)=f\left(x_{0}\right) J_{1, x_{0}}+\mathcal{K}\left[J_{0, x_{0}}, \frac{\partial f_{x_{0}}}{\partial Z_{j}}(0) Z_{j} J_{0, x_{0}}\right] .
\end{gathered}
$$

Here, for any polynomials $F, G \in \mathbb{C}\left[Z, Z^{\prime}\right]$, the polynomial $\mathcal{K}[F, G] \in \mathbb{C}\left[Z, Z^{\prime}\right]$ is defined by the relation

$$
\left(\left(F \mathcal{P}_{x_{0}}\right) \circ\left(G \mathcal{P}_{x_{0}}\right)\right)\left(Z, Z^{\prime}\right)=\left(\mathcal{K}[F, G] \mathcal{P}_{x_{0}}\right)\left(Z, Z^{\prime}\right)
$$

where $\left(\left(F \mathcal{P}_{x_{0}}\right) \circ\left(G \mathcal{P}_{x_{0}}\right)\right)\left(Z, Z^{\prime}\right)$ is the kernel of the composition $\left(F \mathcal{P}_{x_{0}}\right) \circ\left(G \mathcal{P}_{x_{0}}\right)$ of the operators $F \mathcal{P}_{x_{0}}$ and $G \mathcal{P}_{x_{0}}$ in $L^{2}\left(T_{x_{0}} X\right)$ with kernels $\left(F \mathcal{P}_{x_{0}}\right)\left(Z, Z^{\prime}\right)$ and $\left(G \mathcal{P}_{x_{0}}\right)\left(Z, Z^{\prime}\right)$, respectively.

Now we can proceed by a word for word repetition of the corresponding arguments in [24]. So we just give statements of the main results.

First, the following analog of [24, Theorem 6.11] provides a useful criterion for a given family to be a Toeplitz operator.

Theorem 5.12. Let $\left\{T_{p}: L^{2}\left(X, L^{p} \otimes E\right) \longrightarrow L^{2}\left(X, L^{p} \otimes E\right)\right\}$ be a family of bounded linear operators which satisfies the following three conditions:

(i) For any $p \in \mathbb{N}, P_{\mathcal{H}_{p}} T_{p} P_{\mathcal{H}_{p}}=T_{p}$.

(ii) For any $\varepsilon_{0}>0$ and any $l \in \mathbb{N}$, there exists $C_{l, \varepsilon_{0}}>0$ such that for all $p \geqslant 1$ and all $\left(x, x^{\prime}\right) \in X \times X$ with $d\left(x, x^{\prime}\right)>\varepsilon_{0}$,

$$
\left|T_{p}\left(x, x^{\prime}\right)\right| \leqslant C_{l, \varepsilon_{0}} p^{-l} .
$$

(iii) There exists a family of polynomials $\left\{\mathcal{Q}_{r, x_{0}} \in \operatorname{End}(E)_{x_{0}}\left[Z, Z^{\prime}\right]\right\}_{x_{0} \in X}$ such that:

(a) each $\mathcal{Q}_{r, x_{0}}$ has the same parity as $r$,

(b) the family is smooth in $x_{0} \in X$ and

(c) for every $k \in \mathbb{N}$, we have

$$
p^{-n} T_{p, x_{0}}\left(Z, Z^{\prime}\right) \cong \sum_{r=0}^{k}\left(\mathcal{Q}_{r, x_{0}} \mathcal{P}_{x_{0}}\right)\left(\sqrt{p} Z, \sqrt{p} Z^{\prime}\right) p^{-\frac{r}{2}}+\mathcal{O}\left(p^{-\frac{k+1}{2}}\right)
$$

in the sense of (5.26).

Then $\left\{T_{p}\right\}$ is a Toeplitz operator.

Finally, we show that the set of Toeplitz operators on a compact orbifold is closed under the composition of operators, so forms an algebra (an analog of [24, Theorems 6.13 and 6.16]). 
Theorem 5.13. Let $(X, \omega)$ be a compact symplectic orbifold and $\left(L, \nabla^{L}, h^{L}\right)$ be a Hermitian proper orbifold line bundle satisfying the prequantization condition (5.8). Let $\left(E, \nabla^{E}, h^{E}\right)$ be an arbitrary Hermitian proper orbifold vector bundle on $X$.

Given $f, g \in \mathscr{C}^{\infty}(X, \operatorname{End}(E))$, the product of the Toeplitz operators $T_{f, p}$ and $T_{g, p}$ is a Toeplitz operator, more precisely, it admits an asymptotic expansion

$$
T_{f, p} T_{g, p}=\sum_{r=0}^{\infty} p^{-r} T_{C_{r}(f, g), p}+\mathcal{O}\left(p^{-\infty}\right),
$$

where $C_{r}(f, g) \in \mathscr{C}^{\infty}(X, \operatorname{End}(E))$ and $C_{r}$ are bidifferential operators defined locally on each covering $\widetilde{U}$ of an orbifold chart $\left(G_{U}, \widetilde{U}\right) \stackrel{\tau_{U}}{\longrightarrow} U$. In particular $C_{0}(f, g)=f g$.

If $f, g \in \mathscr{C}^{\infty}(X)$, then

$$
\left[T_{f, p}, T_{g, p}\right]=\frac{\sqrt{-1}}{p} T_{\{f, g\}, p}+\mathcal{O}\left(p^{-2}\right) .
$$

For any $f \in \mathscr{C}^{\infty}(X, \operatorname{End}(E))$, the norm of $T_{f, p}$ satisfies

$$
\lim _{p \rightarrow \infty}\left\|T_{f, p}\right\|=\|f\|_{\infty}:=\sup _{\substack{x \in X \\ 0 \neq u \in E_{x}}}|f(x)(u)|_{h^{E}} /|u|_{h^{E}} .
$$

Remark 5.14. As mentioned in [24, Remark 6.14], by [24, Theorems 6.13, 6.16], on every compact symplectic orbifold $X$ admitting a prequantum line bundle $\left(L, \nabla^{L}, h^{L}\right)$, one can define in a canonical way an associative star-product $f * g=\sum_{l=0}^{\infty} \hbar^{l} C_{l}(f, g) \in \mathscr{C}^{\infty}(X)[[\hbar]]$ for every $f, g \in \mathscr{C}^{\infty}(X)$, called the Berezin-Toeplitz star-product by using the kernel of the $\operatorname{spin}^{c}$ Dirac operator. Moreover, $C_{l}(f, g)$ are bidifferential operators defined locally as in the smooth case. Theorem 5.13 shows that one can also use the eigenspaces of small eigenvalues of the renormalized Bochner-Laplacian.

\section{REFERENCES}

[1] A. Adem, J. Leida, and Y. Ruan, Orbifolds and Stringy Topology, Cambridge Tracts in Mathematics, 171, Cambridge University Press, Cambridge, 2007.

[2] J.-M. Bismut and G. Lebeau, Complex immersions and Quillen metrics. Inst. Hautes Études Sci. Publ. Math. No. 74 (1991)

[3] J.-M. Bismut and E. Vasserot, The asymptotics of the Ray-Singer analytic torsion associated with high powers of a positive line bundle, Comm. Math. Phys. 125 (1989), 355-367.

[4] M. Bordemann, E. Meinrenken and M. Schlichenmaier, Toeplitz quantization of Kähler manifolds and $g l(n), n \rightarrow \infty$ limits, Comm. Math. Phys. 165, (1994), 281-296.

[5] D. Borthwick and A. Uribe, Almost complex structures and geometric quantization. Math. Res. Lett. 3 (1996), 845-861.

[6] L. Boutet de Monvel and V. Guillemin, The spectral theory of Toeplitz operators, Ann. of Math. Stud., vol 99, Princeton Univ. Press, Princeton, NJ, 1981.

[7] L. Boutet de Monvel and J. Sjöstrand, Sur la singularité des noyaux de Bergman et de Szegó, Journées: Équations aux Dérivées Partielles de Rennes (1975), pp. 123-164. Astérisque, No. 34-35, Soc. Math. France, Paris, 1976.

[8] D. Catlin, The Bergman kernel and a theorem of Tian, Analysis and Geometry in Several Complex Variables (Katata, 1997), 1-23, Trends Math., Birkhäuser Boston, Boston, MA, 1999.

[9] C. J. Earle, Some remarks on Poincaré series. Compositio Math. 21 (1969) 167-176.

[10] X. Dai, K. Liu, and X. Ma, On the asymptotic expansion of Bergman kernel, J. Differential Geom. 72 (2006), no. $1,1-41$. 
[11] V. Guillemin and A. Uribe, The Laplace operator on the nth tensor power of a line bundle: eigenvalues which are uniformly bounded in $n$. Asymptotic Anal. 1 (1988), 105-113.

[12] L. Ioos, W. Lu, X. Ma and G. Marinescu, Berezin-Toeplitz quantization for eigenstates of the BochnerLaplacian on symplectic manifolds, J. Geom. Anal. (2018). https://doi.org/10.1007/s12220-017-9977-y, preprint arXiv:1703.06420.

[13] C.-Y. Hsiao and G. Marinescu, Berezin-Toeplitz quantization for lower energy forms, Comm. Partial Differential Equations, 42 (2017), 895-942.

[14] Yu. A. Kordyukov, $L^{p}$-theory of elliptic differential operators on manifolds of bounded geometry. Acta Appl. Math. 23 (1991), no. 3, 223-260.

[15] Yu. A. Kordyukov, On asymptotic expansions of generalized Bergman kernels on symplectic manifolds. Algebra i Analiz 30 (2018), no. 2, 163-187; preprint arXiv:1703.04107.

[16] Yu. A. Kordyukov, Semiclassical spectral analysis of Toeplitz operators on symplectic manifolds: The case of discrete wells, preprint arXiv:1809.06799.

[17] W. Lu, X. Ma and G. Marinescu, Donaldson's Q-operators for symplectic manifolds, Sci. China Math. 60 (2017), 1047-1056.

[18] Z. Lu and S. Zelditch, Szegő kernels and Poincaré series. J. Anal. Math. 130 (2016), 167-184.

[19] X. Ma, Orbifolds and analytic torsions, Trans. Amer. Math. Soc. 357 (2005), no. 6, 2205-2233.

[20] X. Ma, Geometric quantization on Kähler and symplectic manifolds. Proceedings of the International Congress of Mathematicians. Volume II, 785-810, Hindustan Book Agency, New Delhi, 2010.

[21] X. Ma and G. Marinescu, The Spin ${ }^{c}$ Dirac operator on high tensor powers of a line bundle. Math. Z. 240 (2002), 651-664.

[22] X. Ma and G. Marinescu, Holomorphic Morse inequalities and Bergman kernels, Progress in Math., vol. 254, Birkhäuser, Basel, 2007, 422 pp.

[23] X. Ma and G. Marinescu, Generalized Bergman kernels on symplectic manifolds. Adv. Math. 217 (2008), $1756-1815$.

[24] X. Ma and G. Marinescu, Toeplitz operators on symplectic manifolds. J. Geom. Anal. 18 (2008), 565-611.

[25] X. Ma and G. Marinescu, Berezin-Toeplitz Quantization and its kernel expansion, Geometry and quantization, 125-166, Trav. Math. 19, Univ. Luxemb., Luxembourg, 2011.

[26] X. Ma and G. Marinescu, Exponential estimate for the asymptotics of Bergman kernels. Math. Ann. 362 (2015), no. 3-4, 1327-1347.

[27] G. Marinescu and N. Savale, Bochner Laplacian and Bergman kernel expansion of semi-positive line bundles on a Riemann surface, preprint arXiv:1811.00992.

[28] J. Milnor, A note on curvature and fundamental group. J. Differential Geom. 2 (1968), 1-7.

[29] G. Tian, On a set of polarized Kähler metrics on algebraic manifolds, J. Differential Geom. 32 (1998), 99-130.

[30] S. Zelditch, Szegő kernels and a theorem of Tian, Int. Math. Res. Not. 6 (1998), 317-331.

Institute of Mathematics with Computing Centre, Ufa Federal Research Centre of Russian AcadEMY OF SCIENCES, 112 CHERNYSHEVSKY STR., 450008 UFA, RUSSIA

E-mail address: yurikor@matem. anrb.ru

Institut de MathÉmatiques de Jussieu-Paris Rive Gauche, UFR de Mathématiques, Université Paris Diderot - Paris 7, CASE 7012, 75205 Paris Cedex 13, France

E-mail address: xiaonan.ma@imj-prg.fr

Universität zu KÖln, Mathematisches Institut, Weyertal 86-90, 50931 KÖln, Germany

Institute of Mathematics 'Simion Stoilow', Romanian ACADEMy, Bucharest, Romania

E-mail address: gmarines@math.uni-koeln.de 